\title{
CYP26C1 Is a Hydroxylase of Multiple Active Retinoids and Interacts with Cellular Retinoic Acid Binding Proteins ${ }^{\mathbf{S}}$
}

\author{
Guo Zhong, David Ortiz, Alex Zelter, Abhinav Nath, and Nina Isoherranen \\ Departments of Pharmaceutics (G.Z., N.I.) and Medicinal Chemistry (D.O., A.N.), School of Pharmacy, and Department of \\ Biochemistry, School of Medicine (A.Z.), University of Washington, Seattle, Washington
}

Received October 31, 2017; accepted February 22, 2018

\section{ABSTRACT}

The clearance of retinoic acid (RA) and its metabolites is believed to be regulated by the CYP26 enzymes, but the specific roles of CYP26A1, CYP26B1, and CYP26C1 in clearing active vitamin A metabolites have not been defined. The goal of this study was to establish the substrate specificity of CYP26C1, and determine whether CYP26C1 interacts with cellular retinoic acid binding proteins (CRABPs). CYP26C1 was found to effectively metabolize all-trans retinoic acid (atRA), 9-cis-retinoic acid (9-cis-RA), 13-cis-retinoic acid, and 4-oxo-atRA with the highest intrinsic clearance toward 9-cis-RA. In comparison with CYP26A1 and CYP26B1, CYP26C1 resulted in a different metabolite profile for retinoids, suggesting differences in the active-site structure of CYP26C1 compared with other CYP26s. Homology modeling of CYP26C1 suggested that this is attributable to the distinct binding orientation of retinoids within the CYP26C1 active site. In comparison with other CYP26 family members, CYP26C1 was up to 10-fold more efficient in clearing 4-oxo-atRA (intrinsic clearance $153 \mu \mathrm{l} / \mathrm{min} / \mathrm{pmol}$ ) than CYP26A1 and CYP26B1, suggesting that CYP26C1 may be important in clearing this active retinoid. In support of this, CRABPs delivered 4-oxo-atRA and atRA for metabolism by CYP26C1. Despite the tight binding of 4-oxo-atRA and atRA with CRABPs, the apparent Michaelis-Menten constant in biological matrix $\left(K_{\mathrm{m}}\right)$ value of these substrates with CYP26C1 was not increased when the substrates were bound with CRABPs, in contrast to what is predicted by free drug hypothesis. Together these findings suggest that CYP26C1 is a 4-oxo-atRA hydroxylase and may be important in regulating the concentrations of this active retinoid in human tissues.

\section{Introduction}

Vitamin A (retinol; ROL), through its active metabolite retinoic acid (RA), plays a critical role in regulation of gene transcription and cell proliferation, differentiation, and apoptosis (Noy, 2010; Tang and Gudas, 2011). Retinol is believed to be devoid of biologic activity and is metabolized by alcohol and aldehyde dehydrogenases to retinoic acid (RA) (Fig. 1; Napoli, 2012; Kedishvili, 2016). The endogenous vitamin A metabolites detected in human serum include all-trans(atRA), 9-cis-, 13-cis-, and 9,13-di-cis-RA, 4-oxo-13-cis-RA, and 4-oxo-atRA (Figs. 1 and 2; Arnold et al., 2012). Of these retinoids, atRA activates retinoic acid receptors (RARs) and is believed to be the most important and biologically active endogenous RA isomer (Allenby et al., 1994; Chambon, 1996; Stevison et al., 2015). However, 13-cis-RA, 9-cis-RA, and 4-oxoatRA can also activate RARs (Idres et al., 2002; Topletz et al., 2015). Owing to this pharmacological activity and their favorable in vivo pharmacokinetics, 13-cis-RA and 9-cis-RA are used to

This research was supported by grants from National Institutes of Health [Grants R01 GM111772 and R01 GM081569].

https://doi.org/10.1124/mol.117.111039.

S This article has supplemental material available at molpharm. aspetjournals.org. treat acne, high risk neuroblastoma (Veal et al., 2007), and chronic hand eczema (Schmitt-Hoffmann et al., 2011). Likewise, both 13-cis-RA and 9-cis-RA are teratogenic, demonstrating classic retinoid effects (Willhite et al., 1986; Kraft and Juchau, 1993). 9-cis-RA is also a ligand of retinoid X receptors (RXRs) with significantly higher binding affinity than atRA and 13-cis-RA (Åström et al., 1990; Heyman et al., 1992; Allenby et al., 1993), but the in vivo significance of 9-cis-RA is unclear. 4-oxo-atRA exhibits higher affinity to $\operatorname{RAR} \alpha$ and similar affinity to $\operatorname{RAR} \beta$ as does atRA (Pijnappel et al., 1993; Idres et al., 2002; Topletz et al., 2015), consistent with its teratogenicity (Herrmann, 1995) and ability to modulate positional specification in early embryo (Pijnappel et al., 1993). However, the role of the different RA isomers and 4-oxoatRA in human physiology is unclear.

Biologic effects of RA isomers and metabolites are dependent on the RAR binding affinity and the cellular concentrations of the retinoids. Therefore, strict regulation of the physiologic concentrations of endogenous retinoids via tissue-specific expression of retinoid-synthesizing and -metabolizing enzymes and retinoid binding proteins (Fig. 1) is critical. Two enzymes of the CYP26 family, CYP26A1 and CYP26B1, have been identified as key enzymes responsible for clearing atRA and controlling atRA concentrations (Fig. 1). CYP26A1 appears to be the liver atRA hydroxylase

ABBREVIATIONS: ACN, acetonitrile; ANOVA, analysis of variance; atRA, all-trans retinoic acid; CRABP, cellular retinoic acid binding protein; EPI, enhanced product ion; FA, formic acid; HPLC, high-performance liquid chromatography; IPTG, isopropyl $\beta$-D-1-thiogalactopyranoside; IS, internal standard; $K_{m}$, apparent Michaelis-Menten constant in biological matrix; LC-MS/MS, liquid chromatography-tandem mass spectrometry; LC-UV, liquid chromatography UV; MD, molecular dynamics; MRM, multiple reaction monitoring; P450, cytochrome P450; RAR, retinoic acid receptor; ROL, retinol. 


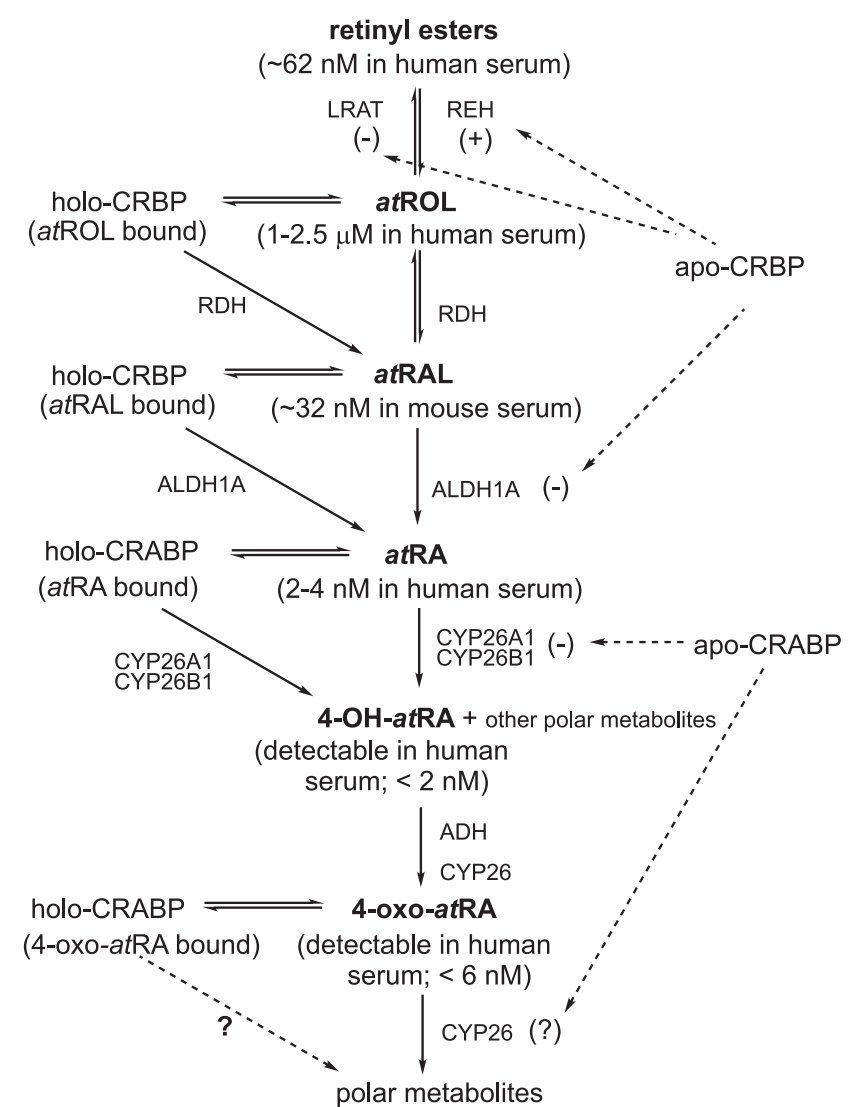

Fig. 1. Enzymes and binding proteins involved in vitamin $A$ and retinoic acid metabolism. Endogenous concentrations of the depicted retinoids in human serum are from prior reports (Bankson et al., 1986; Maqbool et al., 2008; Arnold et al., 2012) and are shown in parenthesis under each retinoid. For alltrans-retinaldehyde (atRAL) no concentrations in human serum have been reported and hence concentrations in mouse serum are provided. The metabolic pathway is adapted from previous publications (Napoli, 2012; Kedishvili, 2016). ADH, alcohol dehydrogenase; ALDH, aldehyde dehydrogenase; CRBP, cellular retinol binding protein; LRAT, lecithin retinol acyltransferase; $\mathrm{RDH}$, retinol dehydrogenase; $\mathrm{REH}$, retinyl ester hydrolase.

eliminating biologically active retinoids, whereas CYP26B1 appears to be responsible for at RA clearance in extrahepatic tissues (Thatcher et al., 2010; Topletz et al., 2015). CYP26A1 and CYP26B1 have also been shown to metabolize 4-oxo-atRA and 9-cis-RA, but the catalytic efficiency is much lower than that toward atRA (Thatcher et al., 2011; Topletz et al., 2012; Diaz et al., 2016), suggesting that other enzymes may be responsible for the elimination of 4-oxo-atRA and RA isomers in humans. The sequence similarity of human CYP26C1 is only $43 \%$ with CYP26A1 and 51\% with CYP26B1 (Taimi et al., 2004), suggesting structural differences between these cytochrome P450s (P450s). Hence, CYP26C1 may have different substrate specificity than CYP26A1 and CYP26B1. Indeed, in CYP26C1-transfected COS-1 cells, both 9-cis-RA and atRA were identified as potential substrates of CYP26C1 (Taimi et al., 2004). However, the role of CYP26C1 in retinoid clearance or in formation of active metabolites such as 4-oxoatRA has not been defined, and biochemical characterization of CYP26C1 in retinoid metabolism is lacking.

A distinct characteristic of biologically important enzymes contributing to retinoid metabolism is that they interact with and obtain their substrates from cellular retinoid binding proteins (Fig. 1; Napoli, 2017). atRA is bound inside cells by

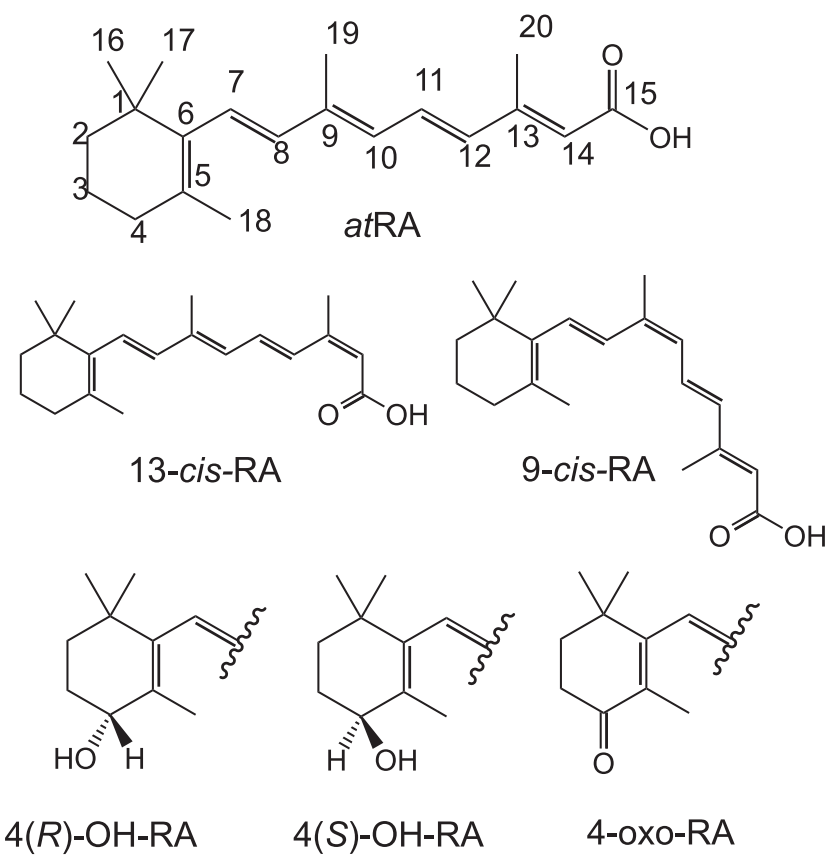

Fig. 2. Chemical structures of RA isomers, 4-OH-RA stereoisomers, and 4-oxo-RA. Numbers in the structure of atRA indicate the convention of numbering for carbon positions.

cellular retinoic acid binding proteins (CRABPs), which appear to deliver atRA either to nuclear RARs to regulate gene transcription or to CYP26A1 and CYP26B1 for catabolism (Napoli, 2017). Hence, whether metabolic enzymes interact with CRABPs can define the biologic importance of the enzyme and the substrates. For example, CRABPI-bound atRA was metabolized in rat testes microsomes at a rate similar to free atRA, and CRABPI-binding of 4-oxo-atRA abolished 4-oxo-atRA metabolism, suggesting substratespecific delivery to metabolism (Fiorella and Napoli, 1991, 1994). Recently, atRA was shown to be channeled to recombinant CYP26B1 by CRABPs (Nelson et al., 2016), but other substrates that bind to CRABPs were not studied, and the specificity of CRABP interactions with CYP26 enzymes was not established. To better understand the role of CYP26 enzymes in retinoid metabolism, the CRABP- and retinoidspecific deliveries of substrates to metabolism by $\mathrm{P} 450$ s need to be defined. The goal of this study was to establish the ligand specificity of CYP26C1 and characterize the CRABPCYP26C1 interactions to delineate the biologic significance and the role of CYP26C1 in regulating retinoid homeostasis.

\section{Materials and Methods}

Chemicals and Reagents. at RA, NADPH, and ketoconazole were purchased from MilliporeSigma (St. Louis, MO). Talarozole was purchased from MedChem Express (Princeton, NJ). 4(S)-OHat RA and $4(R)-\mathrm{OH}$-atRA were synthesized as described previously (Shimshoni et al., 2012). All other retinoids were from Toronto Research Chemicals (North York, ON, Canada). All solvents were high-performance liquid chromatography (HPLC) or Optima grade and were purchased from Thermo Fisher Scientific (Waltham, MA).

Cloning, Expression, and Characterization of Recombinant CYP26C1. Human CYP26C1 cDNA was purchased from OriGene Technologies (Rockville, MD) (SKU: SC307567). Primers were designed to amplify the CYP26C1 coding sequence minus the stop 
codon, and the resulting polymerase chain reaction product was cloned into Invitrogen pFastBac-CT TOPO following the manufacturer's instructions (Thermo Fisher Scientific). The resulting coding sequence consisted of CYP26C1 plus a C-terminal TEV cleavage site followed by a 6xHis-tag. Full-length C-terminal 6xHis-tagged CYP26C1 protein was then produced in Sf9 cells using the Invitrogen Bac-to-Bac baculovirus expression system according to the manufacturer's instructions. Sf-900 II SFM liquid media (Thermo Fisher Scientific, Carlsbad, CA) supplemented with $2.5 \%$ fetal bovine serum was used for cell growth. During protein expression, media was supplemented with $\delta$-aminolevulinic acid $(0.3 \mathrm{mM})$ and ferric citrate $(0.2 \mathrm{mM})$ 24 hours postinfection to enable heme synthesis. Infected cells were harvested after 48 hours, washed in PBS with $1 \mathrm{mM}$ phenylmethylsulfonyl fluoride, and stored at $-80^{\circ} \mathrm{C}$. The microsomal fractions of insect cells were prepared by ultracentrifugation on the basis of published methods (Topletz et al., 2012). The content of active P450 was measured by a CO-difference spectrum (Omura and Sato, 1964), using an Aminco DW-2 dual-beam spectrophotometer (Olis Inc., Bogart, GA). Supersomes containing human P450 oxidoreductase and b5 prepared from insect cells (Corning Inc., Corning, NY) were used as negative control for CO-difference spectra. Protein concentration of the CYP26C1 microsomes was measured using BCA protein assay kit according to manufacturer's recommendations (Thermo Fisher Scientific). The CYP26C1 microsomes were used in all catalytic experiments. Rat P450 reductase was expressed in Escherichia coli and purified as described previously (Woods et al., 2011).

Qualitative Western blotting was conducted on the basis of a published method for CYP26A1 with minor modifications (Lutz et al., 2009). CYP26C1 microsomes containing $2 \mu \mathrm{g}$ total protein were loaded on the gel. Mouse anti-6xHis antibody (1:2000; Qiagen, Valencia, CA) and IRDye 680RD anti-mouse antibody (1: 10000; LI-COR Biosciences, Lincoln, NE) were used as primary and secondary antibodies, respectively, to detect the His-tagged CYP26C1.

General Protocol for Incubations. Unless otherwise described, incubations and retinoid extractions were performed following published procedures used for analysis of CYP26A1 and CYP26B1 activity (Thatcher et al., 2010; Topletz et al., 2012). Similar incubation conditions and reductase to $\mathrm{P} 450$ ratios were used for consistency. The rat reductase was added into CYP26C1 microsomes and allowed to incorporate into the membranes at room temperature for 5 minutes (Thatcher et al., 2010; Topletz et al., 2012), before membranes were added to the incubation mixture containing substrate and $100 \mathrm{mM}$ potassium phosphate buffer in a total volume of $1 \mathrm{ml}$. After another 5 minutes' preincubation at $37^{\circ} \mathrm{C}$ with the substrate, the reaction was initiated with the addition of $1 \mathrm{mM}$ NADPH. Incubations were quenched and extracted with $3 \mathrm{ml}$ of ethyl acetate, as previously described (Topletz et al., 2012). The ethyl acetate layer was collected, evaporated to dryness under $\mathrm{N}_{2}$ flow, and reconstituted with $100 \mu \mathrm{l}$ of methanol. Incubation time, microsomal CYP26C1 content and rat reductase concentration used for incubations are specified for each experiment below. 4-Oxo-atRA-d $\mathrm{d}_{3}(2 \mu \mathrm{l}$ of $5 \mu \mathrm{M}$ solution) was added as an internal standard (IS) for all the incubations except those using UV-Vis detection. All product formation was confirmed to be NADPHdependent by comparison to identical incubations in the absence of NADPH. Incubations were conducted in duplicate or triplicate and all experiments repeated three times.

Evaluation of Potential CYP26C1 Substrates. To identify substrates of CYP26C1, individual retinoids at $1 \mu \mathrm{M}$ concentration were incubated with $18 \mathrm{nM}$ microsomal CYP26C1 and $36 \mathrm{nM}$ rat reductase for 30 minutes. Tested retinoids included RA isomers (atRA, 13-cis-RA, and 9-cis-RA), 4-oxo-RA isomers (4-oxo-atRA, 4-oxo-13-cis-RA, and 4-oxo-9-cis-RA), 4-OH-RA isomers (4-OH-atRA, 4-OH-13-cis-RA, and 4-OH-9-cis-RA), ROL isomers (atROL, 13-cisROL, and 9-cis-ROL), and 3-dehydro-atROL. For incubations with RA isomers, 4-oxo-RA isomers, or 4-OH-RA isomers, samples were extracted and analyzed by liquid chromatography (LC)-UV as described previously (Thatcher et al., 2010) using an Agilent 1200 series HPLC system (Agilent Technologies, Santa Clara, CA) equipped with a Zorbax C18 column (3.5 $\mu \mathrm{m}, 2.1 \times 100 \mathrm{~mm}$; Agilent Technologies) and by using water instead of $50 \mathrm{mM}$ ammonium acetate $(\mathrm{pH} 4.5)$ in the mobile phase. The column temperature was maintained at room temperature. Analytes were monitored at UV wavelength of $360 \mathrm{~nm}$. For incubations with ROL isomers and 3-dehydro-atROL, hexanes $(4 \mathrm{ml})$ were used to quench the reaction and extract analytes. After the hexane layer was collected and evaporated, samples were reconstituted with $100 \mu \mathrm{l}$ acetonitrile (ACN). Retinyl acetate $(5 \mu \mathrm{l}$ of $5 \mu \mathrm{M}$ solution) was added to the samples as IS. Samples were analyzed by LC-UV using the Agilent 1200 series HPLC system coupled with an Ascentis RP-amide column $(2.7 \mu \mathrm{m}, 15 \mathrm{~cm} \times 2.1 \mathrm{~mm}$; MilliporeSigma $)$. Column temperature was maintained at $40^{\circ} \mathrm{C}$. The flow rate was $0.5 \mathrm{ml} / \mathrm{min}$. The gradient elution started with initial condition of $40 \%$ aqueous with $0.1 \%$ formic acid (FA) and $60 \%$ acetonitrile with $0.1 \%$ FA, which was maintained for 2 minutes, followed by a gradient to $100 \%$ ACN with $0.1 \%$ FA over 11 minutes, and held at that for 8 minutes before being returned to the initial conditions before next injection. Analytes were monitored at UV wavelength of $325 \mathrm{~nm}$.

Stereoselective Formation of 4-OH-RA Enantiomers from RA Isomers by CYP26C1. The stereoselectivity in the formation of 4-OH-RA by CYP26C1 was determined for individual RA isomers by incubating atRA, 13-cis-RA, or 9-cis-RA at $0.75 \mu \mathrm{M}$ with $15 \mathrm{nM}$ CYP26C1 and $30 \mathrm{nM}$ reductase for 15 minutes. For comparison, CYP26A1 was incubated under identical conditions with the same substrates. Samples were extracted and analyzed by LC-UV using an Agilent 1200 series HPLC system coupled with a Chiralcel OD-RH column ( $5 \mu \mathrm{m}, 2.1 \times 150 \mathrm{~mm}$; Chiral Technologies Inc., West Chester, $\mathrm{PA})$ as described previously to detect $4(S)$ - and $4(R)$-OH-RA formation (Fig. 2; Shimshoni et al., 2012). The peak area ratio of $4(S)$ - and $4(R)$ $\mathrm{OH}-\mathrm{RA}$ was calculated and used as an indicator of the stereoselectivity of 4-OH-RA formation.

Identification of Metabolites Formed by CYP26C1. To identify metabolites formed by CYP26C1, the incubations described above for substrate identification were further analyzed by liquid chromatography-tandem mass spectrometry (LC-MS/MS) using an Sciex API5500 Q/LIT mass spectrometer (Sciex, Concord, ON, Canada) equipped with Agilent 1290 Infinity UHPLC (Agilent Technologies). All analytes were monitored with electrospray ionization under negative ion mode. To evaluate the identity of metabolites of RA isomers, samples were first separated using a Zorbax C18 column (3.5 $\mu \mathrm{m}, 2.1 \times 100 \mathrm{~mm}$ ) and a mobile phase flow rate of $0.35 \mathrm{ml} / \mathrm{min}$, with a linear gradient from $90 \%$ aqueous with $0.1 \% \mathrm{FA}$ and $10 \% \mathrm{ACN}$ to $5 \%$ aqueous with $0.1 \% \mathrm{FA}$ and $95 \% \mathrm{ACN}$ over 10 minutes. The column temperature was maintained at $40^{\circ} \mathrm{C}$. The multiple-reaction monitoring (MRM) transitions included $\mathrm{m} / z 315>253$ (4-OH- and 18-OHRA), $m / z 315>241$ (16-OH-RA), and $m / z 313>269$ (4-oxo-RA), as described previously (Topletz et al., 2012). All MS parameters set for MRM scans are listed in Supplemental Table 1. Together with monitoring MRM transitions, parallel information of $[\mathrm{M}-\mathrm{H}]$ ions, including $\mathrm{m} / z 315$ and $\mathrm{m} / z 313 \mathrm{Da}$, were acquired via enhanced product ion (EPI) scan. The MS/MS spectra were collected from $\mathrm{m} / z$ 50-330 Da. EPI scan rate was set at 10,000 Da/s, linear ion trap fill time at 100 milliseconds, $Q_{1}$ at low resolution and $Q_{3}$ entry barrier at $8 \mathrm{~V} . \mathrm{Q}_{0}$ trap was turned on. Other mass spectrometer detection settings were the same as those used in MRM scan, except that declustering potential (DP) of $-80 \mathrm{~V}$ and collision energy (CE) of $35 \mathrm{~V}$ with a spread of $\pm 10 \mathrm{~V}$ for both $[\mathrm{M}-\mathrm{H}]$ ions was used. For further separation of the metabolites, the MS/MS spectra were also collected after chiral separation of the products using the Chiralcel OD-RH column and chromatography as described above. Mass spectrometry settings for MRM and EPI scans were as described above.

To determine the identity of 4-oxo-atRA metabolites, a Zorbax C18 column was used for analyte separation, and the incubations were analyzed by LC-MS/MS. The flow rate was $0.2 \mathrm{ml} / \mathrm{min}$, with a linear gradient from $90 \%$ aqueous with $0.1 \% \mathrm{FA}$ and $10 \% \mathrm{ACN}$ to $10 \%$ aqueous with $0.1 \% \mathrm{FA}$ and $90 \% \mathrm{ACN}$ over 15 minutes, held for 8 minutes before being returned to initial conditions. The MRM transitions monitored included $\mathrm{m} / z 329>255$ (4-oxo-16-OH-atRA) 
and $m / z 329>267 \mathrm{Da}$ (4-oxo-18-OH- $a t$ RA), as described previously (Topletz et al., 2012). Parameters set for MRM scans are provided in Supplemental Table 1. The EPI scan with the spectrum range of 50350 Da was acquired for the [M-H] ion of $m / z 329$ Da. EPI settings were similar to those described for metabolites of RA isomers except that the scan rate was $1000 \mathrm{Da} / \mathrm{S}$ and $\mathrm{Q}_{1}$ was set at unit resolution.

Enzyme Kinetics of RA Isomers and 4-oxo-atRA with CYP26C1. To determine enzyme kinetic parameters for RA isomers, varying concentrations of atRA $(10-250 \mathrm{nM}), 13-c i s-\mathrm{RA}(5-250 \mathrm{nM})$, or 9-cis-RA (3-100 nM) were incubated with $2 \mathrm{nM} \mathrm{CYP26C1}$ and $4 \mathrm{nM}$ reductase for 30 seconds. Standard curves of RA isomers, 4-OH-RA isomers, and 4-oxo-RA isomers were constructed for quantification. Samples were extracted and analyzed by LC-MS/MS using Sciex QTRAP 4500 mass spectrometer (Sciex) coupled with Shimadzu UFLC XR DGU-20A5 (Shimadzu Corporation, Kyoto, Japan) and a Zorbax C18 column, as described above for metabolite identification. 4-OH-, 18-OH- and 16-OH-RA isomers, 4-oxo-RA isomers, 4-oxo-atRA$\mathrm{d}_{3}$, and RA isomers were monitored by MRM, and the MRM transitions and MS parameters used are listed in Supplemental Table 2. On the basis of the peak area ratio of metabolites to the IS, the formation of major metabolites of RA isomers was quantified using Analyst software (Sciex). Owing to the sequential metabolism of 4-OH-RA to 4-oxo-RA, to quantify the 4-OH-RA formation from atRA and 13-cis-RA, the formation of 4-OH-RA and 4-oxo-RA were summed, as previously described for CYP26A1 and CYP26B1 (Topletz et al., 2015) (Supplemental Fig. 1A). For 9-cis-RA, only the formation of 4-OH-9-cis-RA was quantified because the formation of 4-oxo-9-cis-RA was quantitatively insignificant. The velocity of metabolite formation was plotted as a function of RA concentration, and substrate concentration was corrected for depletion by calculating the average of the initial added concentration and final measured concentration, as described previously (Lutz et al., 2009). Enzyme kinetic parameters, including $k_{\text {cat }}$ and apparent Michaelis-Menten constant in biological matrix $\left(K_{\mathrm{m}}\right)$, were obtained from GraphPad Prism 5.0 (GraphPad Software, La Jolla, CA) by fitting the Michaelis-Menten equation to the data. Intrinsic clearance $\left(C l_{\text {int }}\right)$ was calculated as $k_{\text {cat }} / K_{\mathrm{m}}$.

To obtain enzyme kinetic parameters for 4-oxo-atRA, both product formation and substrate depletion of 4-oxo-atRA by CYP26C1 were measured. For product formation, varying concentrations of 4-oxoatRA (3-100 nM) were incubated with $2 \mathrm{nM} \mathrm{CYP26C1}$ and $4 \mathrm{nM}$ reductase for 2 minutes. Samples were extracted and analyzed by LC-MS/MS using Sciex 4500 QTRAP mass spectrometer and the Zorbax C18 column with the same LC conditions as described above. Monitored analytes included 4-oxo-16-OH-atRA, 4-oxo-atRA, and 4-oxo-atRA- $\mathrm{d}_{3}$. MRM transitions and MS parameters are listed in Supplemental Table 2. Owing to the tight binding of 4-oxo-atRA to CYP26C1, enzyme kinetic parameters, including $k_{\text {cat }}$ and $K_{\mathrm{m}}$, were obtained from GraphPad Prism 5.0 by fitting the Morrison equation to the data, as described previously (Shimshoni et al., 2012), and assuming a CYP26C1 concentration of $2 \mathrm{nM}$ in the incubations. Because of the lack of synthetic 4-oxo-16-OH-atRA standard, the product formation was estimated as the ratio of peak area of 4-oxo-16OH-atRA to IS to allow $K_{\mathrm{m}}$ determination. For substrate depletion, experiments were conducted following published procedures (Shimshoni et al., 2012) with varying concentrations of 4-oxo-atRA $(5-150 \mathrm{nM})$ incubated with $2 \mathrm{nM}$ CYP26C1 and $4 \mathrm{nM}$ reductase in a total volume of $3 \mathrm{ml}$. At $0.5,1,2$, and 4 minutes, $0.5-\mathrm{ml}$ aliquots were taken and quenched with $3 \mathrm{ml}$ of ethyl acetate. 4-oxo-atRA- $\mathrm{d}_{3}$ was added as an IS and 4-oxo-atRA quantified as described for product formation assays. $K_{\mathrm{m}}$ and $C l_{\text {int }}$ were obtained using GraphPad Prism 5.0, as described previously (Shimshoni et al., 2012).

Differences in the kinetic parameters between substrates were evaluated using Graphpad Prism 5.0. The differences for each kinetic parameter $\left(K_{\mathrm{m}}, k_{\text {cat }}\right.$, and $\left.C l_{\text {int }}\right)$ were tested via analysis of variance (ANOVA), including all different substrates in the analysis, and Tukey's test was used as a post-hoc test to identify differences between substrates. Owing to the multiple ANOVA comparisons done (comparison of $K_{\mathrm{m}}, k_{\text {cat }}$, and $C l_{\text {int }}$ values), a Bonferroni adjustment for the
$P$ value was done $(0.05 / 3)$ and a $P$ value less than 0.01 was considered significant.

Expression and Purification of CRABPs. CRABP expression vectors were a gift from Dr. Noa Noy (Case Western Reserve University). His-tagged CRABP-I and CRABP-II vectors were used to transform E. coli. Transformed cells were plated into LB agar plates containing $30 \mu \mathrm{g} / \mathrm{ml}$ of kanamycin. Overnight cultures were prepared from freshly streaked plates. LB media $(500 \mathrm{ml})$ with kanamycin added was inoculated with overnight cultures and incubated at $37^{\circ} \mathrm{C}$ with shaking until optical density at the wavelength of $600 \mathrm{~nm}$ reached 0.6. IPTG was added at a final $1 \mathrm{mM}$ concentration and the culture was incubated at $37^{\circ} \mathrm{C}$ for another 2 hours. Induced cells were collected by centrifugation at $6000 \mathrm{~g}$ for 20 minutes. Cell pellets were stored at $-80^{\circ} \mathrm{C}$ until protein purification. For purification of CRABPs, frozen cell pellets were thawn on ice, resuspended with $20 \mathrm{ml}$ of lysis buffer $(1 \mathrm{mg} / \mathrm{ml}$ of lysozyme, $500 \mathrm{mM} \mathrm{NaCl}, 20 \mathrm{mM}$ Tris, $5 \mathrm{mM}$ imidazole, pH7.4), and incubated at room temperature for 20 minutes with gentle shaking. Resuspended mixture was placed on ice and sonicated for 30 seconds twice with a 30 -second interval followed by centrifugation at $12,000 \mathrm{~g}$ for 30 minutes. Supernatant was applied to a HisTrap HP column (GE Healthcare Bio-Sciences, Marlborough, MA) using Biologic DuoFlow chromatography system (Bio-Rad Laboratories, Hercules, CA) following manufacturer's instructions. Loaded samples were first washed with $5 \times$ column volume of $20 \mathrm{mM}$ Tris- $\mathrm{HCl}, 500 \mathrm{mM} \mathrm{NaCl}, 20 \mathrm{mM}$ imidazole (pH 7.4), and then CRABPs were eluted with $5 \times$ column volume of $20 \mathrm{mM}$ Tris- $\mathrm{HCl}, 500 \mathrm{mM} \mathrm{NaCl}, 300 \mathrm{mM}$ imidazole ( $\mathrm{pH}$ 7.4). Fractions containing CRABPs were concentrated and exchanged into HEDK buffer $(10 \mathrm{mM}$ Hepes, $0.1 \mathrm{mM}$ EDTA, $0.5 \mathrm{mM}$ DTT, $100 \mathrm{mM} \mathrm{KCl}$, pH 8.0) using Amicon $10-\mathrm{kD}$ molecular-weight cutoff filters (MilliporeSigma, Billerica, MA). Purified CRABPs were stored at $-20^{\circ} \mathrm{C}$ in HEDK buffer with $50 \%$ glycerol.

Effect of CRABPs on Retinoid Metabolism by CYP26C1. To investigate how CRABPs affect atRA, 9-cis-RA, and 4-oxo-atRA metabolism by CYP26C1, three experiments were conducted as previously described with minor modifications (Nelson et al., 2016). For all the CRABP-related incubations, microsomal CYP26C1 $(2 \mathrm{nM})$ and rat reductase $(4 \mathrm{nM})$ were first preincubated with $1 \mathrm{mM}$ NADPH in a total volume of $1 \mathrm{ml}$ at $37^{\circ} \mathrm{C}$ for 3 minutes. The reaction was initiated by adding free substrate (atRA, 4-oxo-atRA, or 9-cis-RA) or substrates premixed with CRABP-I or CRABP-II in 1:1 concentration ratio. Oneto-one binding of substrates (atRA or 4-oxo-atRA) with CRABPs was confirmed with fluorescence titration and via isolation of the holoCRABP from CRABP-ligand mixture using the method adapted from Fiorella et al. (Fiorella and Napoli, 1991; Fiorella et al., 1993) in which the ligand is bound with CRABP and then holo-CRABP is separated from any free ligand using a mini-desalting column (Thermo Fisher Scientific). After the incubation time specified for each experiment, the reaction was quenched with $\mathrm{ACN}(1 \mathrm{ml})$ followed by the addition of ethyl acetate $(3 \mathrm{ml})$ and $4-0 x 0-a t \mathrm{RA}-\mathrm{d}_{3}(2 \mu \mathrm{l}$ of $5 \mu \mathrm{M}$ solution), as previously described (Nelson et al., 2016). The rest of the procedures were as described above and metabolite formation was measured as described above using LC-MS/MS. To characterize the effect of CRABPs on CYP26C1 activity, first, CYP26C1 was incubated with $100 \mathrm{nM}$ free substrate or $100 \mathrm{nM}$ CRABP-ligand mixture (1:1) for 2 minutes. The formation rates of 4-OH-atRA, 4-OH-9-cis-RA, and 4-oxo-16-OH-atRA were compared in the absence and presence of CRABPs. Second, the kinetics of 4-OH-atRA and 4-oxo-16-OH-atRA formation as metabolites of atRA and 4-oxo-atRA were characterized, respectively. For this, varying concentrations of CRABP-I and CRABP-II-bound atRA or 4-oxo-atRA (3-200 nM; 1:1 CRABP-ligand ratio) were incubated with CYP26C1 for 1 (at RA) or 2 minutes (4-oxoatRA) and extracted as described above. The $K_{\mathrm{m}}$ and $k_{\text {cat }}$ of atRA and 4-oxo-atRA bound to CRABP were obtained by fitting the MichaelisMenten equation and the Morrison equation to the data, respectively. Third, the effect of excess apo-CRABPs on CYP26C1 activity was evaluated as described previously for CRABPs and CYP26B1 (Nelson et al., 2016). Individual substrate at $100 \mathrm{nM}$ was premixed with $0-400$ nM CRABP-I or CRABP-II and incubated with CYP26C1 in the 
presence of NADPH for 2 minutes. Metabolite formation rates were measured and compared with the control, which contained no CRABP in the incubation. Differences in kinetic values were compared together with all substrates by ANOVA followed by Tukey's test as a post-hoc test as described above for enzyme kinetic analysis.

Ligand-Induced Binding Spectra and Determination of $\mathrm{IC}_{50}$ Values of Talarozole and Ketoconazole with CYP26C1. To explore the active-site characteristics of CYP26C1 and test if talarozole and ketoconazole bind to the active site of CYP26C1 and interact with the heme, CYP26C1 microsomes at $0.7 \mu \mathrm{M}$ in $100 \mathrm{mM}$ potassium phosphate buffer ( $\mathrm{pH} 7.4$ ) were added into the sample and reference cuvettes, with $0.45 \mathrm{ml}$ in each. Microsomal CYP26C1 was titrated with ketoconazole $(100,149$, and $198 \mu \mathrm{M})$ or talarozole $(20,79$, and $157 \mu \mathrm{M})$ in methanol in the sample cuvette, and an equal volume of methanol was added into the reference cuvette. The binding spectra were obtained using an Aminco DW2 dual-beam spectrophotometer (Olis Inc.). The spectra were normalized to 415 or $420 \mathrm{~nm}$ for comparison.

To determine if talarozole and ketoconazole are also inhibitors of CYP26C1 and to obtain $\mathrm{IC}_{50}$ values, talarozole $(0.01-25 \mu \mathrm{M}$ dissolved in dimethyl sulfoxide) and ketoconazole (25-200 $\mu \mathrm{M}$ dissolved in methanol) were incubated with $2 \mathrm{nM}$ CYP26C1, $4 \mathrm{nM}$ reductase, and $50 \mathrm{nM}$ atRA for 2 minutes. Because the $K_{\mathrm{m}}$ values for 9-cis-RA were much lower than atRA with CYP26C1, instead of using 9-cis-RA as a substrate in the inhibition assays, as previously described (Thatcher et al., 2011), assays were performed using atRA as a substrate at $50 \mathrm{nM}$, a concentration similar to the $K_{\mathrm{m}}$ value of atRA. Control incubation contained a volume of dimethyl sulfoxide or methanol equal to those used with talarozole or ketoconazole. Samples were analyzed by LC-MS/MS as described above for enzyme kinetic experiments. The peak area ratio of 4-OH-atRA to IS was used as a measure of CYP26C1 activity. The percent remaining activity compared with control was calculated and plotted as a function of inhibitor concentration. The $\mathrm{IC}_{50}$ values were determined by nonlinear regression using GraphPad Prism 5.0, as described previously (Thatcher et al., 2011).

Generation of a CYP26C1 Homology Model and Docking of Retinoids. To explore the ligand binding interactions of CYP26C1, a CYP26C1 homology model was created with I-TASSER (Zhang Lab, University of Michigan, Ann Arbor, MI; Roy et al., 2010), using the sequence for human CYP26C1 (NCBI sequence number NP_899230.2). The following structures were used as templates: the retinoic acid-bound cyanobacterial CYP120A1 (PDB code 2VE3; Kühnel et al., 2008); the lanosterol- (PDB code 4LXJ), itraconazole(PDB code 5EQB; Monk et al., 2014), and fluconazole- (PDB code 4WMZ; Sagatova et al., 2015) bound structures of a CYP51 from Saccharomyces cerevisiae; and the thioperamide-bound human CYP46A1 (PDB code 3MDM; Mast et al., 2010). The heme group was positioned in I-TASSER models according to its position in structural analogs. To model a structure in solution capable of binding CYP26 substrates, molecular dynamics (MD) simulations were performed with GROMACS version 4.6.5 (Van Der Spoel et al., 2005). The topology files were modified to reflect the cysteine thiolate linkage to the heme iron, as previously described (Autenrieth et al., 2004; Oda et al., 2005). Atomic interactions were defined with the GROMOS 54a7 force field. The model was placed in a dodecahedron periodic box of diameter $3.75 \mathrm{~nm}$ and then energy was minimized by the steepestdescent and conjugate-gradient methods. The system was solvated with $\sim 89,000$ simple point-charge water molecules, and $\mathrm{Na}^{+}$and $\mathrm{Cl}^{-}$ ions were added to neutralize the system with an effective $0.15 \mathrm{M}$ ionic strength. Molecular dynamics simulations reached equilibrium in $\sim 7$ nanoseconds and were allowed to proceed for 40 nanoseconds. Rootmean-square deviation and active-site volume during the MD simulation are shown in Supplemental Fig. 2. To select a conformation suitable for docking studies, the active-site volume throughout the MD simulation was measured with POcket Volume MEasurer 2.0 (POVME; Durrant et al., 2014). Candidate models were selected from different time points representing conformations with maximum, minimum, and intermediate active-site volumes. Each candidate was then subjected to in silico ligand docking screens in Autodock 4.0 (Österberg et al., 2002). The heme charges were manually edited to match the high-spin ferric state, as described previously (Shahrokh et al., 2012). The following ligands were obtained from PubChem and docked to each candidate model: all-trans-RA (compound identifier CID 444795), 9-cis-RA (CID 449171), 13-cis-RA (CID 5282379), and 4-oxo-atRA (CID 6437063). Side-chains from the following residues were selected to be flexible during the docking simulations for all substrates: Trp-117, Phe-295, Phe-299, and Ile-497. Additionally, Leu131 was made flexible for the docking of 4-oxo-atRA, and Thr-300 was made flexible for the docking of 9-cis-RA and 4-oxo-atRA. Initial docking screens were performed with 10 simulations per ligand and $2.5 \times 10^{5}$ energy evaluations per docking simulation. The model derived from the 8-nanosecond time point has an active-site volume that reaches a local maximum (Supplemental Fig. 2B) and permits the binding of each ligand with high affinity. This model was chosen as the most suitable candidate. The final CYP26C1 homology model was subjected to more extensive docking studies of 200 simulations per ligand with the same parameters as above. The quality of the model before and after refinement was validated by Ramachandran plot analysis (RAMPAGE server; Lovell et al., 2003) and Verify3D score (Eisenberg et al., 1997). Parameters of homology model quality assessment and the secondary structure assignments are listed in Supplemental Tables 3 and 4.

\section{Results}

Expression and Characterization of CYP26C1. Microsomal CYP26C1 prepared from baculovirus-infected Sf9 insect cells showed a typical CO-difference spectrum with a defined peak at $448 \mathrm{~nm}$, which was not observed in the negative control (Fig. 3). The P450 content, calculated from the CO-difference spectrum and from the microsomal protein content, was $84.3 \mathrm{pmol} / \mathrm{mg}$ of protein $(2.2 \mu \mathrm{M})$ in the membrane preparation. The Western blot developed with anti$6 \mathrm{xHis}$ antibody indicated expression of a protein with an approximate size of 50-60 kD, corresponding to the predicted molecular weight of CYP26C1, $57 \mathrm{kD}$ (Fig. 3), demonstrating CYP26C1 expression in Sf9 insect cell microsomes.

Identification and Characterization of CYP26C1 Substrates. Among all tested retinoids, all three RA isomers, 4-OH-RA isomers, and 4-oxo-atRA were substrates of CYP26C1, showing significant metabolite formation in the incubations with CYP26C1 on the basis of UV absorbance (Figs. 4-6). In contrast, no significant metabolite formation
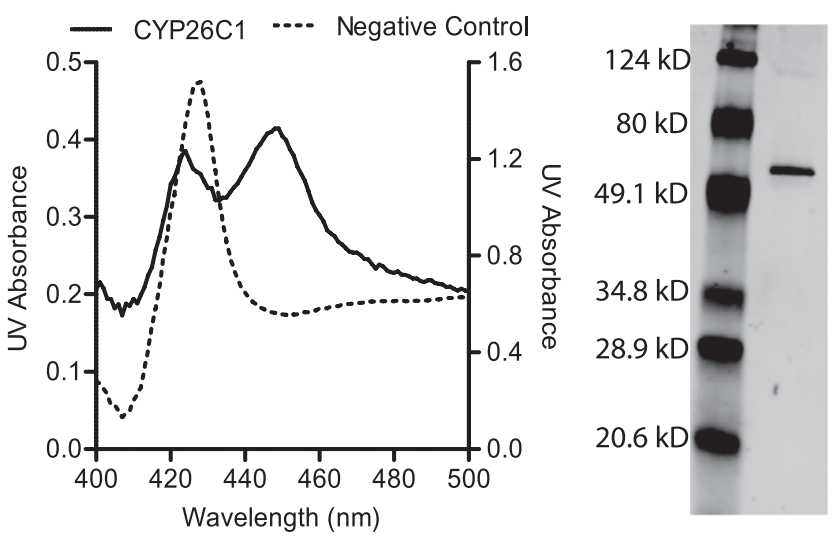

Fig. 3. CO-difference spectrum and western blot of microsomal CYP26C1. In CO-difference spectrum, the left and right $Y$-axis are for microsomal CYP26C1 and negative control, respectively. 
was detected in incubations with retinol isomers, 3-dehydro-atROL (Supplemental Fig. 3), 4-oxo-13-cis-RA (Fig. 6B), or 4-oxo-9-cis-RA (Fig. 6C). With all RA isomers (atRA, 13-cis-RA, or 9-cis-RA), the 4-hydroxylation pathway was the primary metabolic pathway resulting in formation of 4-OH-RA isomers, and subsequent minor formation of 4-oxo-RA and other hydroxylation products (M1-M8). The primary hydroxylation products at the $\mathrm{C} 16$ and $\mathrm{C} 18$ positions were observed from atRA, and C16 hydroxylation was observed from 13-cis-RA and 9-cis-RA (Fig. 4). The identity of these minor metabolites was confirmed by LC-MS/MS (Fig. 4, G-I). Notably, the polar metabolites (M1-M8) observed in the incubations with atRA, 13-cis-RA and 9-cis-RA (Fig. 4) corresponded exactly to those observed as products from the incubations of the corresponding 4-OH-RA isomers after incubation with CYP26C1 (Fig. 5), suggesting sequential metabolism of 4-OH-RA isomers formed from RA by CYP26C1. For 4-oxo-RA isomers, CYP26C1 metabolized 4-oxo-atRA to a single metabolite identified as 4-oxo16-OH-atRA by LC-MS/MS (Fig. 6, A and D), but metabolism of
4-oxo-13cisRA and 4-oxo-9-cis-RA was negligible (Fig. 6). The identity of the metabolites formed by CYP26C1 was confirmed via collecting MS/MS spectra (Supplemental Figs. 4-6). The fragmentation patterns of metabolites were consistent with those described in previous studies with other CYP26s (Thatcher et al., 2011; Topletz et al., 2012).

To further define the metabolite pattern for CYP26C1, the stereospecificity of the formation of 4-OH-RA (Fig. 2) from atRA, 13-cis-RA, or 9-cis-RA by CYP26C1 was evaluated using chiral chromatography (Fig. 7). In the incubation with atRA, $4(R)-\mathrm{OH}-a t \mathrm{RA}$ was the main enantiomer produced by CYP26C1, with a $4 \mathrm{~S} / 4 \mathrm{R}$ ratio of 0.2 (Fig. $7 \mathrm{G}$ ). In comparison, similar amounts of $4(S)$ - and $4(R)$-OH-13-cis-RA were formed by CYP26C1 with a 4S/4R ratio of 1.3 (Fig. 7H). From 9-cis-RA only $4(S)$-OH-9-cis-RA was detected, demonstrating distinct stereospecificity in 4-OH-RA formation (Fig. 7I). The stereospecificity of 4-OH-RA formation was clearly different for CYP26A1 in comparison with CYP26C1. CYP26A1 produced mainly $4(S)$-OH-RA from all three RA isomers. The $4 \mathrm{~S} / 4 \mathrm{R}$

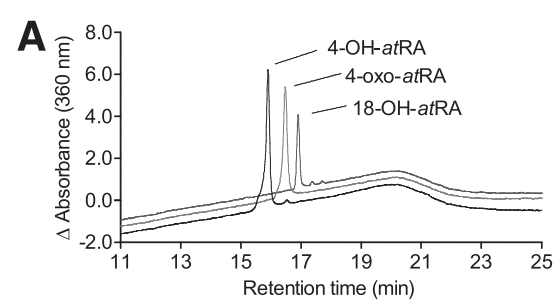

D
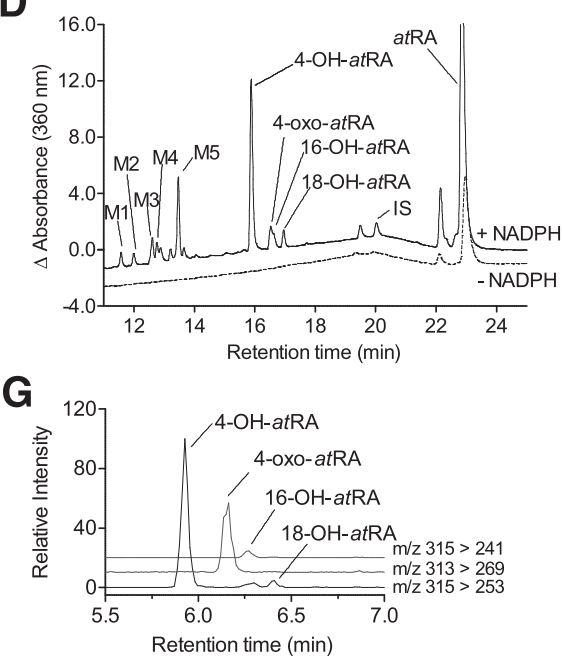

$\mathbf{L}$

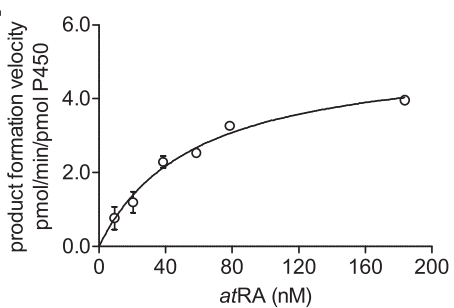

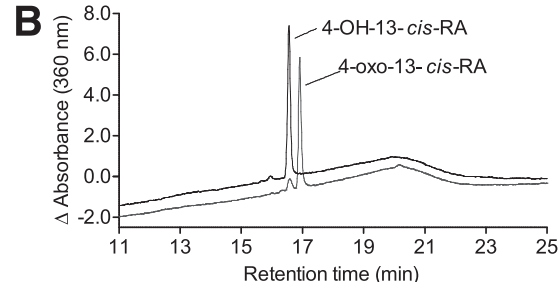

E

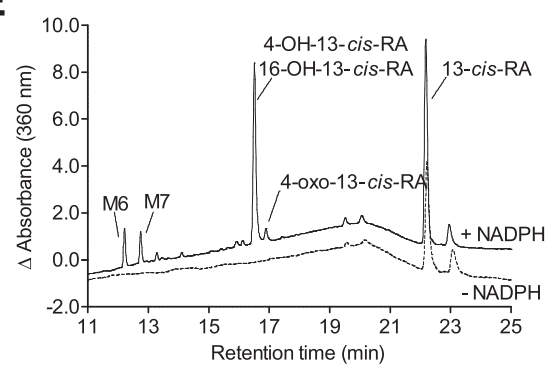

H

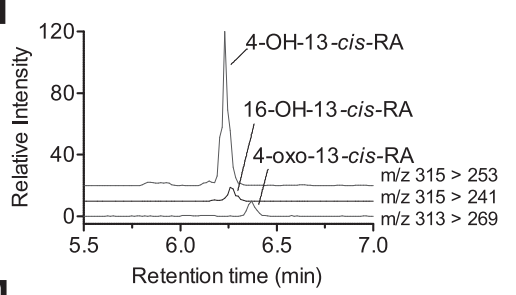

M

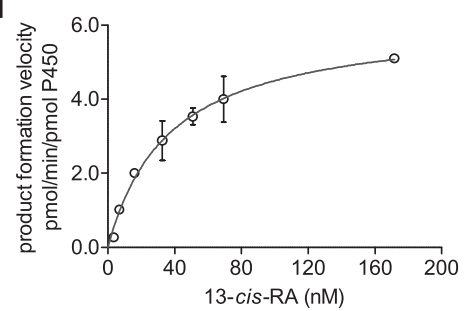

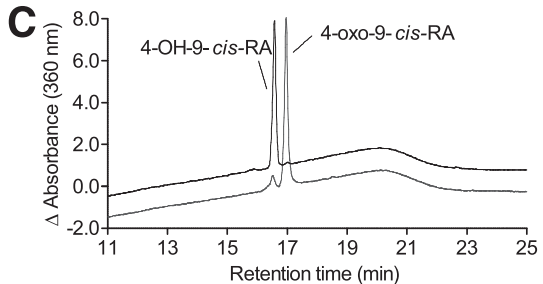

$\mathbf{F}$

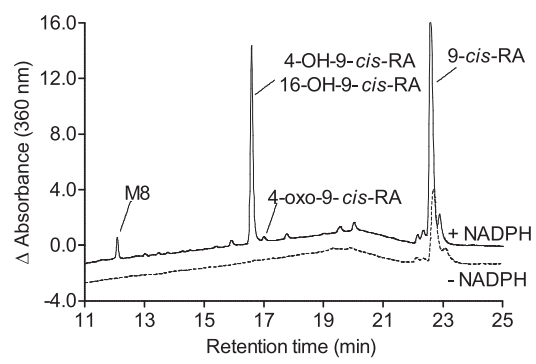

I

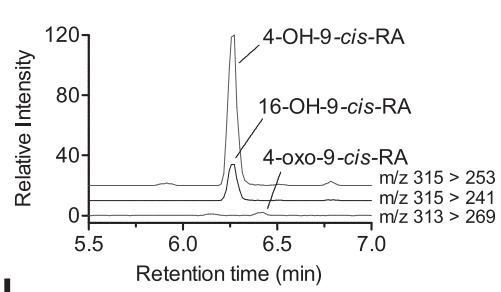

$\mathbf{N}$

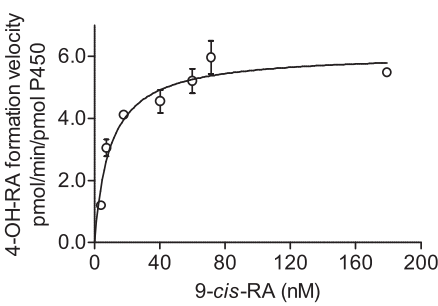

Fig. 4. Characterization of the metabolism of RA isomers by CYP26C1. From left to right, CYP26C1 was incubated with atRA, 13-cis-RA, and 9-cis-RA, respectively. (A-C) LC-UV chromatograms of synthetic standards. Middle panels are UV (D-F) and LC-MS/MS chromatograms (G-I) of metabolite formation by CYP26C1. Indication of 16-OH-RA formation in LC-UV chromatograms (D-F) was based on MRM chromatograms (G-I) and EPI scan (Supplemental Figs. 4-6) despite the lack of 16-OH-RA standards. (L-N) Representative Michaelis-Menten plots from three repeated experiments for each substrate for the formation of the primary metabolites. To quantify the 4-OH-RA formation from atRA (L) and 13-cis-RA (M), the formation of 4-OH-RA and 4-oxo-RA were summed (Supplemental Fig. 1A). For 9-cis-RA (N), only the formation of 4-OH-9-cis-RA was quantified because the formation of 4-oxo-9-cis-RA was quantitatively insignificant. The data points shown are the mean values of duplicate measurements with error bars as range. Each kinetic experiment was repeated three times to obtain kinetic value estimates (Table 1). 

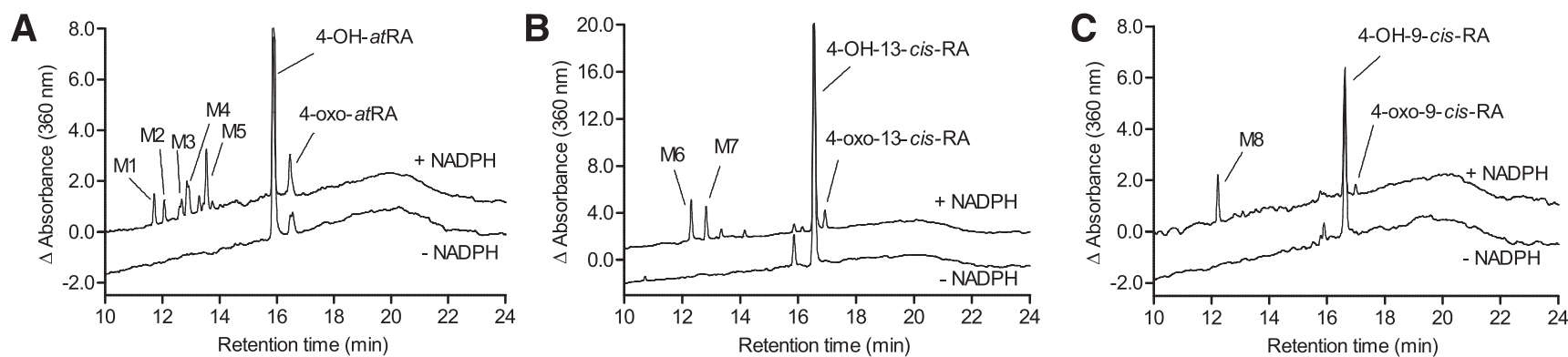

Fig. 5. Metabolism of 4-OH-RA isomers by CYP26C1. LC-UV chromatograms of 4-OH-atRA (A), 4-OH-13-cis-RA (B), and 4-OH-9-cis-RA (C) metabolism by CYP26C1 are shown in the presence and absence of NADPH. Sequential metabolites are labeled as M1-M8 corresponding to the numbering in Fig. 4, D-F.

ratios were 5.5 and 7.6 with atRA and 13-cis-RA as CYP26A1 substrates, respectively (Fig. 7, D and E). With 9-cis-RA as the substrate, only the peak of $4(S)-\mathrm{OH}-9$-cis-RA was observed with CYP26A1 (Fig. 7F).

In addition to the separation of the 4-OH-RA enantiomers, the separation of 16-OH-13-cis-RA from 4-OH-13-cis-RA and 16-OH-9-cis-RA from 4-OH-9-cis-RA was much better when chiral chromatography (Supplemental Figs. 4-6) was used, rather than standard reversed phase separation (Fig. 4, E and F), allowing confirmation of the formation of these metabolites and collection of good quality MS/MS spectra of the metabolites of 13-cis-RA and 9-cis-RA (Supplemental Figs. 5 and 6). The metabolites identified included the hydroxylations at $\mathrm{C} 4$ and $\mathrm{C} 16$ positions and sequential formation of the 4-oxometabolites.

Enzyme Kinetic Parameters of RA Isomers and 4-oxo-atRA as Substrates of CYP26C1. The $k_{\text {cat }}$ values with CYP26C1 for all RA isomers were similar, whereas $K_{\mathrm{m}}$ values of atRA and 13-cis-RA were 5.1- and 1.6-fold higher than those of 9-cis-RA, respectively (Table 1 ). The $C l_{\text {int }}$ value for 9-cis-RA was 4.2- and 2.4-fold higher than that for atRA and 13-cis-RA, respectively. This suggests that CYP26C1 prefers 9-cis-RA as a substrate over atRA and 13-cis-RA (Fig. 4, L-N; Table 1). The relatively robust formation of the 16-OH- metabolite from 9-cis-RA allowed the determination of the $K_{\mathrm{m}}$ value toward this metabolite formation $(5.9 \pm 0.3 \mathrm{nM}$; mean \pm S.D., $n=3$; Supplemental Fig. 1B), as well as for 4-OH-9-cis-RA formation, and the $K_{\mathrm{m}}$ values for the two metabolites were similar (Table 1 ). In the case of 4-oxo-atRA as a substrate of CYP26C1, similar $K_{\mathrm{m}}$ values were obtained by measuring product formation and substrate depletion $(P>$ 0.01 ; Fig. $6, \mathrm{E}$ and F; Table 1 ). These $K_{\mathrm{m}}$ values were similar to those of 9-cis-RA but significantly lower than those of atRA and 13-cis-RA $(P<0.01$; Table1), suggesting that 4-oxo-atRA has higher affinity to CYP26C1 than atRA and 13-cis-RA, and CYP26C1 may prefer 4-oxo-atRA as a substrate. Because of
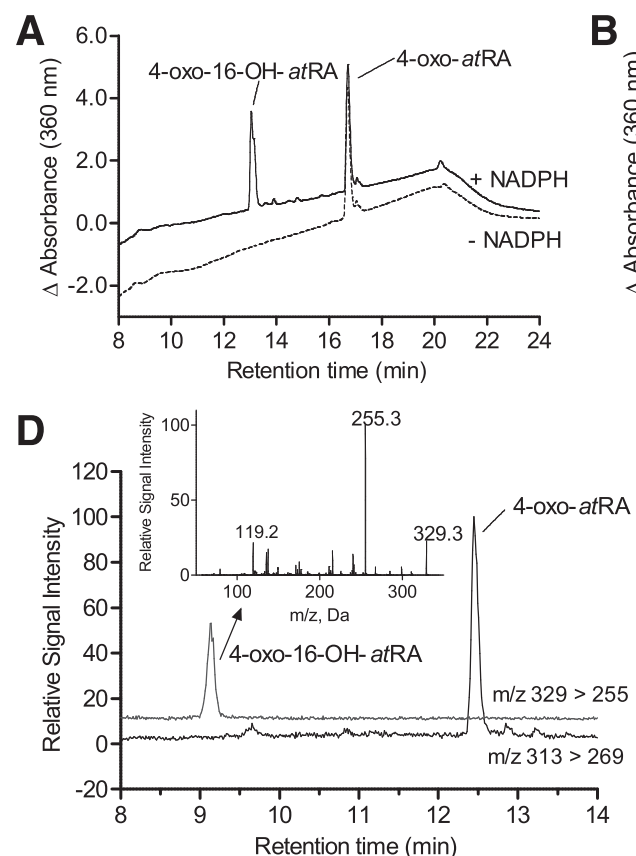

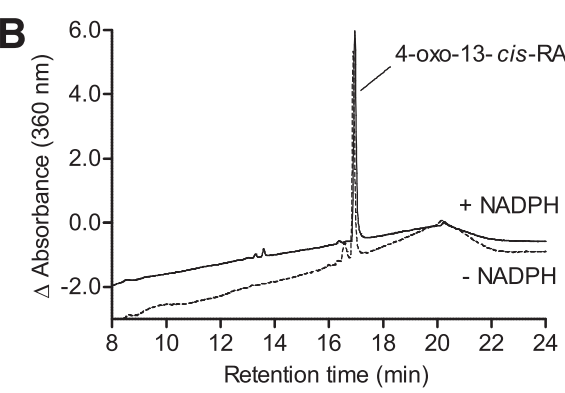

E

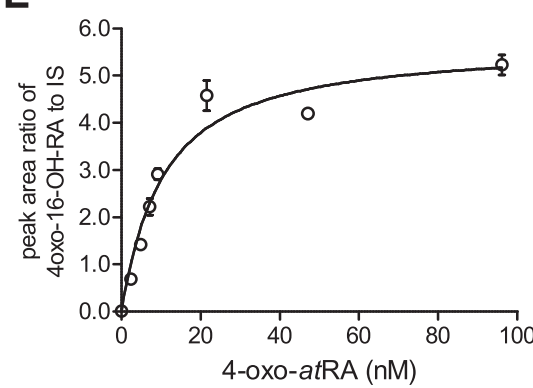

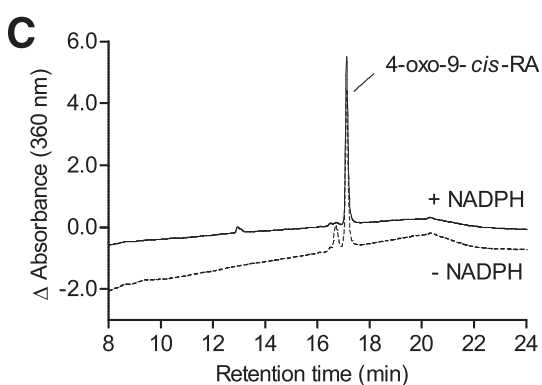

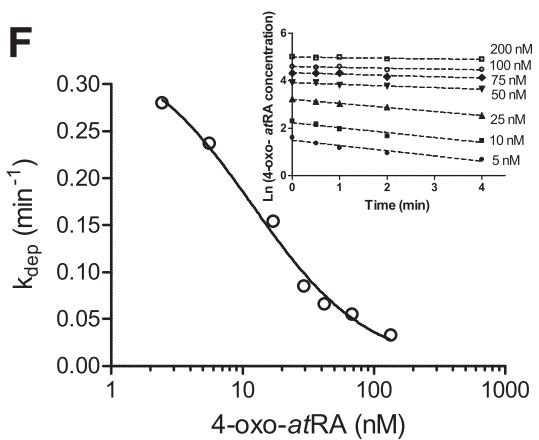

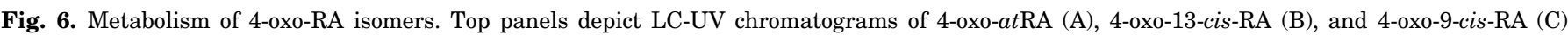

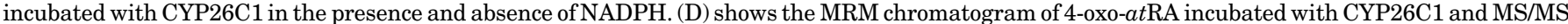

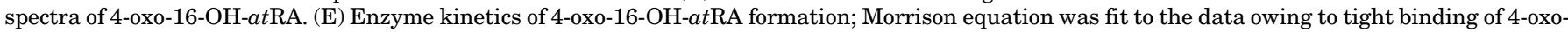

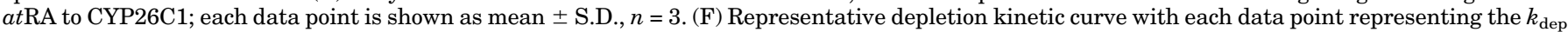
value obtained from the depletion experiment (inset). 

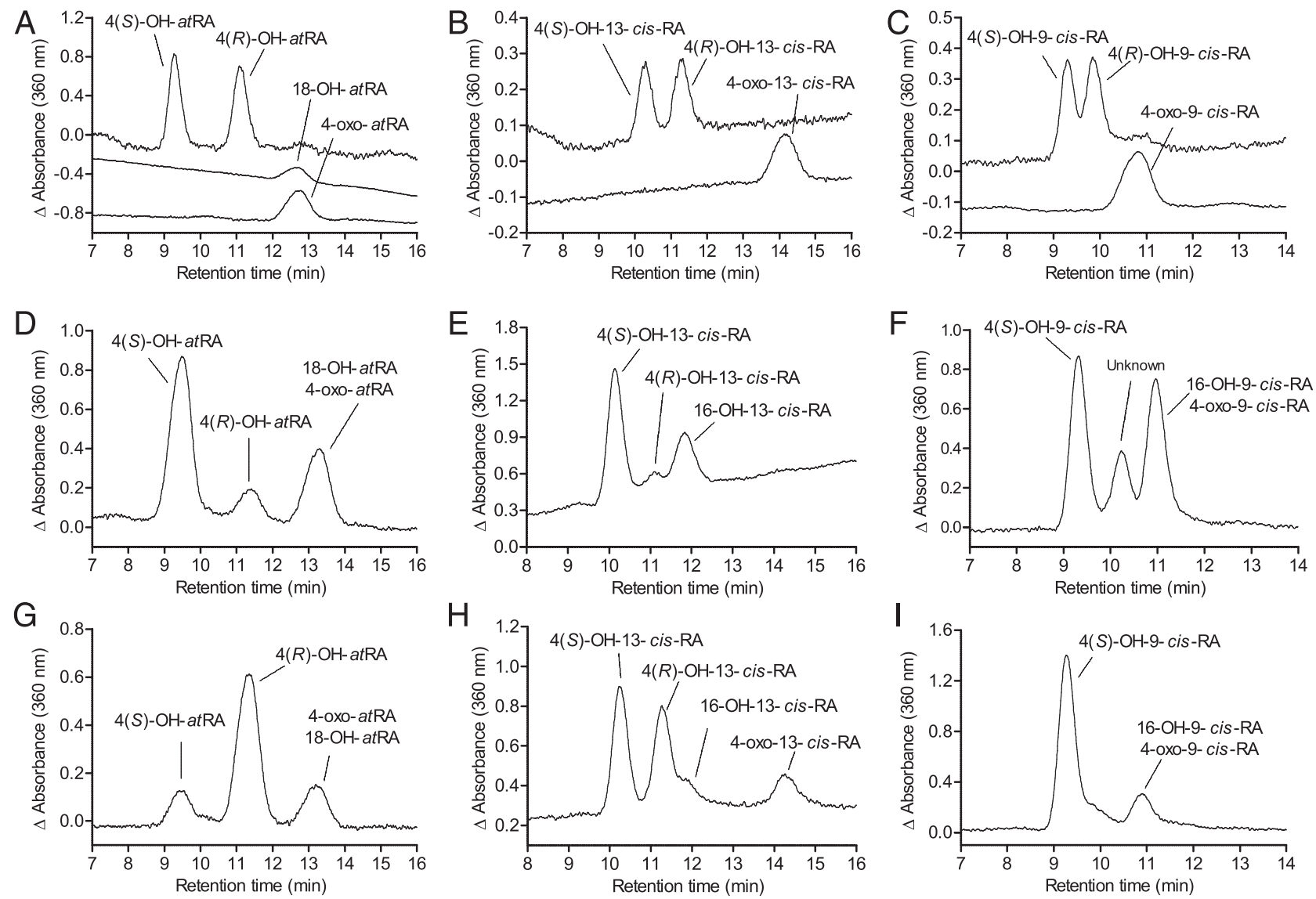

Fig. 7. Stereoselective formation of 4-OH-RA enantiomers by CYP26A1 (D-F) and CYP26C1 (G-I). From left to right, CYP26 was incubated with atRA, 13-cis-RA, and 9-cis-RA, respectively. (A-C) LC-UV chromatograms of synthetic standards.

the lack of synthetic 4-oxo-16-OH-atRA standard, the $k_{\text {cat }}$ for product formation was not measured but the efficiency of 4-oxo-atRA metabolism was determined via substrate depletion (Fig. 6F). Of all the substrates, 9-cis-RA had the highest $C l_{\text {int }}$ by CYP26C1, which was significantly greater (3.3-fold, $P<0.01$; Table 1) than that of 4-oxo-atRA, whereas the $C l_{\text {int }}$ of atRA, 13-cis-RA, and 4-oxo-atRA were not significantly different from each other $(P>0.01$; Table 1$)$.

Effect of CRABPs on Metabolism by CYP26C1. Since retinoids are extensively bound to CRABPs in cellular environment, the delivery of CRABP-bound substrates to metabolic enzymes has often been used as an indicator of the

TABLE 1

Enzyme kinetic parameters of RA isomers, 4-oxo-atRA, CRABP-bound atRA, and CRABP-bound 4-oxoat RA as substrates with CYP26C1

Data are shown as mean \pm S.D. All means are average values of three replicate experiments with each experimenta datapoint determined in triplicate in each experiment. Differences in the kinetic parameters $\left(k_{\text {cat }}, K_{\mathrm{m}}\right.$, and $\left.\mathrm{Cl}_{\text {int }}\right)$ were analyzed by one-way ANOVAs (separate ANOVA for each parameter) followed by Tukey's multiple comparison test. Owing to the multiple ANOVA analyses, a $P$ value of 0.01 was considered significant on the basis of a Bonferroni adjustment for multiple comparisons.

\begin{tabular}{|c|c|c|c|}
\hline & $k_{\text {cat }}$ & $K_{\mathrm{m}}$ & $C l_{\text {int }}$ \\
\hline & $\min ^{-1}$ & $n M$ & $\mu l /$ min per picomolar $P 450$ \\
\hline$a t \mathrm{RA}$ & $5.9 \pm 1.9$ & $50.4 \pm 6.2$ & $120 \pm 49$ \\
\hline 13-cis-RA & $7.7 \pm 2.9$ & $38.2 \pm 4.9$ & $208 \pm 101$ \\
\hline 9-cis-RA & $4.7 \pm 1.2$ & $9.7 \pm 2.6^{c d}$ & $504 \pm 140^{f}$ \\
\hline 4-oxo-at $\mathrm{RA}^{a}$ & & $15.7 \pm 4.3^{c^{c} d}$ & $153 \pm 20$ \\
\hline 4-oxo- $a t \mathrm{RA}^{b}$ & & $10.2 \pm 2.2^{c, d}$ & \\
\hline CRABPI- $a t$ RA & $2.1 \pm 0.2$ & $50.8 \pm 8.0^{e}$ & $41 \pm 2$ \\
\hline CRABPII- $a t$ RA & $2.1 \pm 0.8$ & $34.1 \pm 7.1^{e}$ & $66 \pm 37$ \\
\hline CRABPI-4oxo- $a t \mathrm{RA}$ & & $3.5 \pm 0.5^{c}$, & \\
\hline CRABPII-40xo- $a t$ RA & & $5.4 \pm 1.5^{c} d$ & \\
\hline
\end{tabular}

${ }^{a}$ Kinetic parameters were obtained by measuring substrate depletion.

${ }^{b} K_{\mathrm{m}}$ value was obtained by measuring product formation.

${ }^{c} P<0.01$, significantly different from the $K_{\mathrm{m}}$ value of at RA.

${ }^{d} P<0.01$, significantly different from the $K_{\mathrm{m}}$ value of 13 -cis-RA.

${ }^{e} P<0.01$, significantly different from the $K_{\mathrm{m}}$ value of 9-cis-RA.

$f_{P}<0.01$, the $\mathrm{Cl}_{\text {int }}$ value of 9 -cis-RA is significantly different from the other $C l_{\text {int }}$ values. 
biologic importance of a metabolic enzyme in retinoid homeostasis. In addition, the delivery of atRA for metabolism by P450s has been shown to be enzyme-dependent (Nelson et al., 2016). For example, binding of atRA to CRABP-I and CRABP-II abolished the metabolism of atRA by CYP3A4 and CYP2C8 (Nelson et al., 2016), as predicted by the free drug hypothesis, i.e., that only drug free in solution and not protein-bound is available for metabolism. However, CRABP-I and CRABP-II delivered atRA for metabolism by CYP26B1 (Nelson et al., 2016). On the other hand, atRA binding to albumin resulted in reduced metabolism of atRA by CYP26B1, as predicted by free drug hypothesis and by albumin acting as a binding sink of atRA (Nelson et al., 2016). To investigate whether CYP26C1 takes any of its substrates from CRABP-I or CRABP-II, and interacts with the binding proteins, CYP26C1 was incubated with CRABP-bound atRA, 4-oxo-atRA, and
9-cis-RA (CRABP-ligand ratio of $1: 1$; Fig. 8A). In the case of atRA, compared with the no CRABP incubations, 4-OH-atRA formation was decreased by $60 \%-70 \%$ in the presence of CRABP-I or CRABP-II (Fig. 8A). However, no change of 4-OH-9-cis-RA formation by CYP26C1 was observed in the presence of CRABPs when 9-cis-RA was the substrate (Fig. 8A). With 4-oxo-atRA as a substrate, 4-oxo-atRA metabolism by CYP26C1 was about $75 \%$ and $45 \%$ lower in the presence of CRABP-I and CRABP-II, respectively, in comparison with the incubations with 4-oxo-atRA as a substrate in the absence of the CRABPs (Fig. 8A).

To further investigate the interactions between CRABPs and CYP26C1 with different CYP26C1 substrates, the kinetics of metabolite formation were characterized using CRABPbound substrates. In kinetic studies with CYP26C1 (Fig. 8B; Table 1 ), the $k_{\text {cat }}$ of atRA was $64 \%$ lower in the presence of

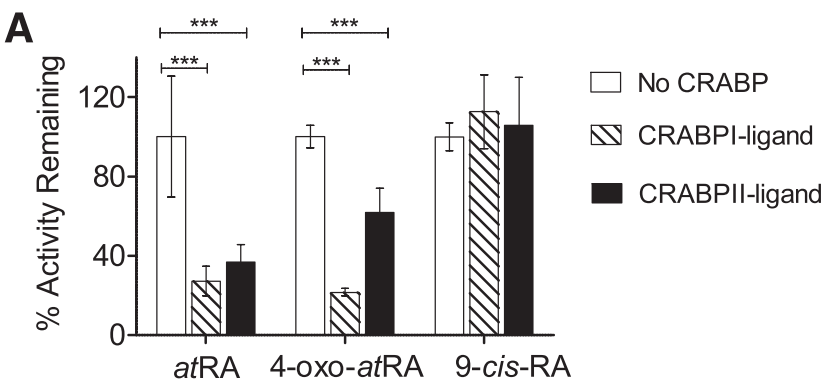

B
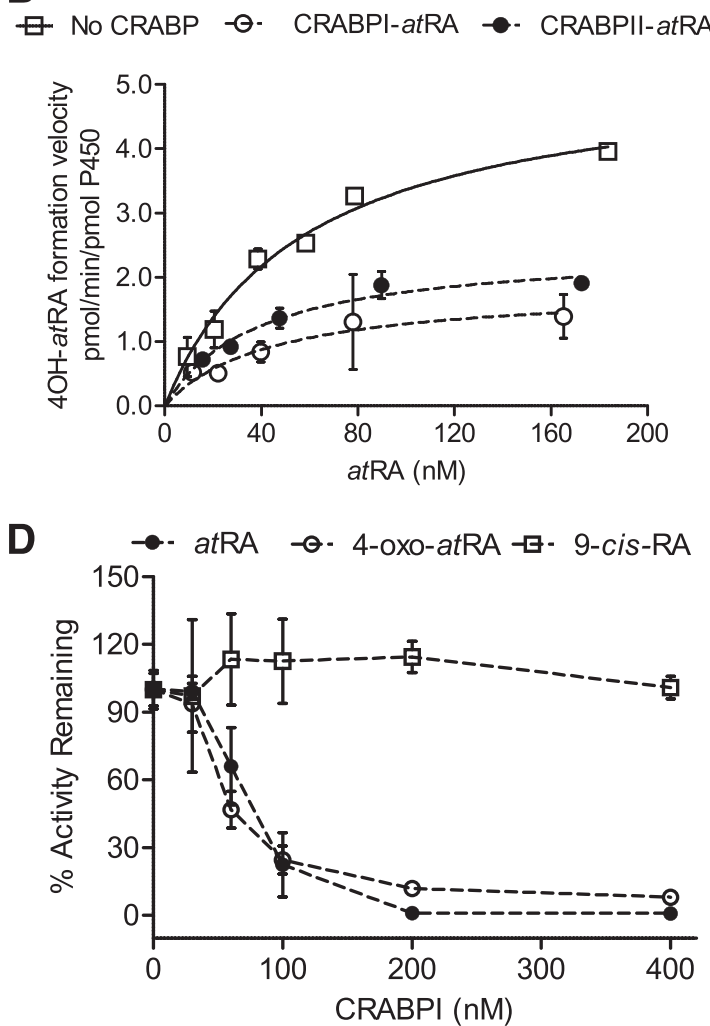

C ๑ No CRABP $-\odot \cdot$ CRABPI-40xo $\rightarrow \cdot$ CRABPII-40xo

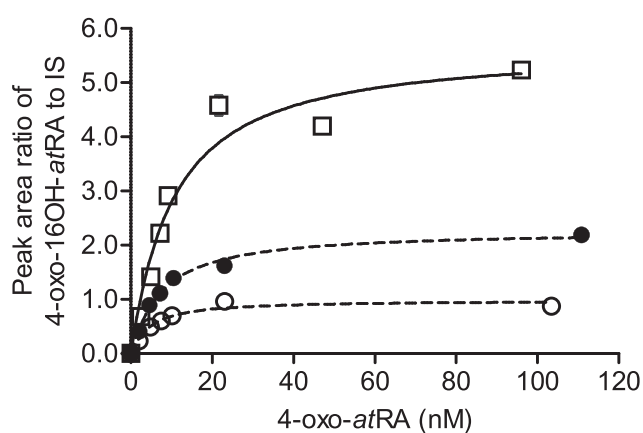

E

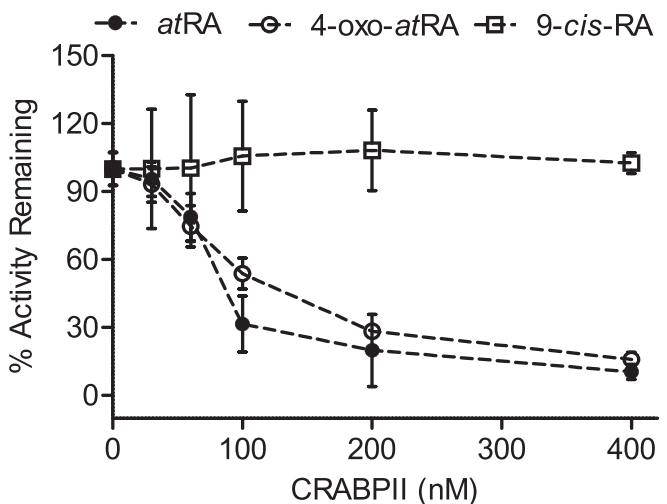

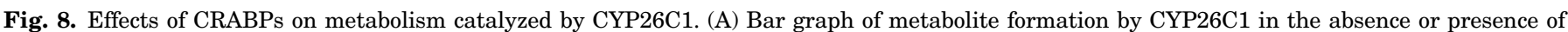

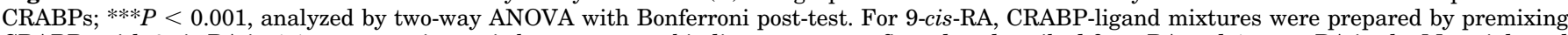

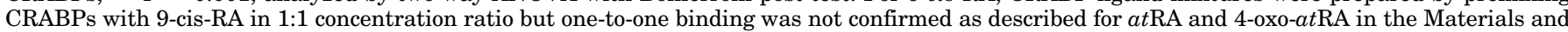

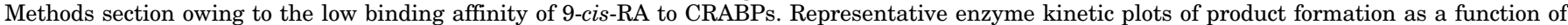

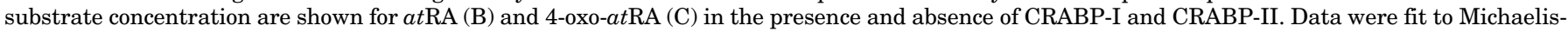

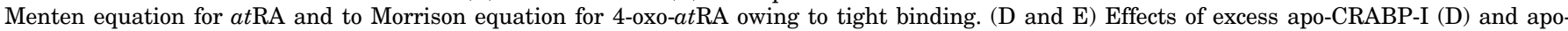
CRABP-II (E) on product formation from atRA, 4-oxo-atRA, and 9-cis-RA (100 nM for each substrate). 
CRABP-I and CRABP-II compared with free atRA, but this difference was not statistically significant. The $K_{\mathrm{m}}$ value of CRABP-I- $a t$ RA and CRABP-II- $a t$ RA remained unchanged compared with that of free atRA $(P>0.01$; Table 1$)$. As atRA binds to CRABPs tightly, virtually no unbound atRA is expected to be present in the incubations, including CRABPs. Therefore, this data suggests that CRABPs deliver atRA to CYP26C1. Although there was a trend toward decreased intrinsic clearance of atRA in the presence of CRABPs, the intrinsic clearances were not significantly different $(P>0.01$; Table 1) in the presence and absence of CRABPs. CRABP-I and CRABP-II affected 4-oxo-atRA metabolism in a manner similar to atRA (Fig. 8C; Table 1 ). The $K_{\mathrm{m}}$ values for 4oxoatRA obtained in the presence of CRABP-I and CRABP-II were similar to those obtained in the absence of CRABPs $(P>0.01$; Table 1$)$. The estimated maximum peak area ratio of 4-oxo-16OH-atRA to the IS obtained in the presence of CRABP-I and CRABP-II (Fig. 8C), which is an indicator of the $k_{\text {cat }}$, was reduced about $80 \%$ and $60 \%$, respectively, compared with that obtained in the absence of CRABPs, suggesting a similar decrease in the intrinsic clearance of 4-oxo- $16 \mathrm{OH}$ atRA formation. However, owing to the lack of reference standard for this metabolite, the true $k_{\text {cat }}$ values could not be quantified.

To study the effect of excess apo-CRABPs on product formation by CYP26C1, a fixed concentration of substrate was mixed with varying concentrations of CRABP-I and CRABP-II, with the ratio of CRABP to substrate ranging from 0 to 4 (Fig. 8, D and E). In the case of atRA and 4-oxo-atRA, CYP26C1 activity decreased as the ratio of CRABP to substrate increased, reaching a plateau after the ratio exceeded 1 . This suggests that apo-CRABPs do not compete with holo-CRABPs to interact with CYP26C1, but CRABPs may decrease metabolism by CYP26C1 via allosteric modulation or via noncompetitive inhibition. Surprisingly, neither CRABP-I nor CRABP-II had any effect on 9-cis-RA metabolism and there was no significant change in 4-OH-9-cis-RA formation from 9-cis-RA by CYP26C1 as the concentration of CRABP-I or CRABP-II increased from 0 to $400 \mathrm{nM}$. This further suggests that CRABP-P450 interactions are substrate-dependent.

Binding Spectra and $\mathrm{IC}_{50}$ Values of Talarozole and Ketoconazole. The substrate specificity and stereoselectivity of the product formation with CYP26C1 suggests that CYP26C1 has different ligand interactions than do other members of the CYP26 family. To explore the ligand specificity of CYP26C1, the binding of the two azole inhibitors of CYP26A1 and CYP26B1, ketoconazole, and talarozole, were tested with CYP26C1. The coordination of the triazole or imidazole nitrogen with the heme iron causes a high-spinto-low-spin shift of the iron, resulting in a type 2 binding spectrum. The binding of ketoconazole or talarozole to microsomal CYP26C1 induced a type 2 binding spectrum with some atypical characteristics, with the minimum absorbance observed between 410 and $420 \mathrm{~nm}$ and the maximum absorbance between 420 and $430 \mathrm{~nm}$ (Fig. 9, A and B). This suggests that the azole nitrogen in these two inhibitors can coordinate with the CYP26C1 heme. The binding was inhibitor concentration-dependent and showed increased spectral intensity with increasing inhibitor concentration. In agreement with the binding spectra, both ketoconazole
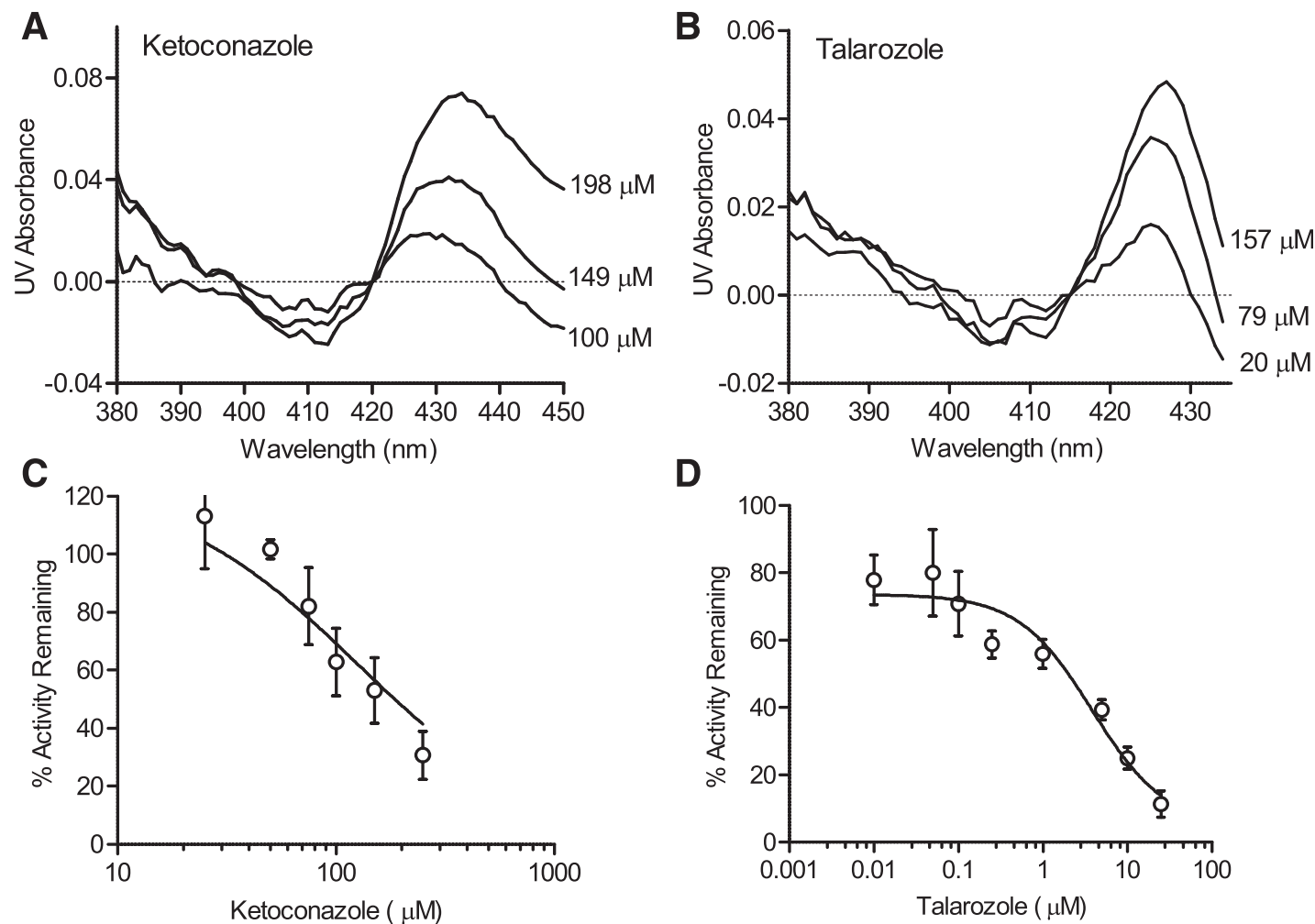

D

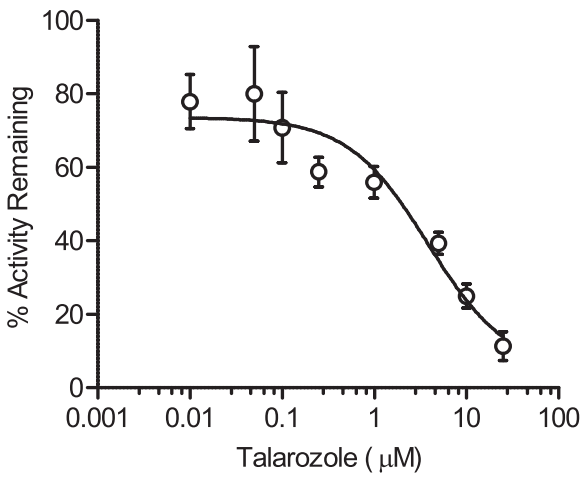

Fig. 9. Characterization of the inhibition of CYP26C1 by imidazole- and triazole-containing inhibitors. Binding spectra of ketoconazole (A) and talarozole (B) with CYP26C1, and the inhibitor concentration-dependent decrease in CYP26C1 activity with ketoconazole (C) and talarozole (D). Inhibitor concentrations are indicated in binding spectra. Data points in (C) and (D) are shown as mean \pm S.D., $n=4$. For fitting of the $\mathrm{IC}_{50}$ values, the $y$-intercept was constrained as less than $125 \%$ and the minimum activity remaining as greater than 0 . 
and talarozole inhibited CYP26C1 activity in an inhibitor concentration-dependent manner (Fig. 9, C and D). However, the $\mathrm{IC}_{50}$ values, $124 \mu \mathrm{M}$ (26-594; 95\% confidence intervals) with ketoconazole and $3.8 \mu \mathrm{M}(1.8-8.1 ; 95 \% \mathrm{CI})$ with talarozole, were much higher than the $\mathrm{IC}_{50}$ values observed with CYP26A1 ( $<10 \mathrm{nM}$ for talarozole and $0.55 \mu \mathrm{M}$ for ketoconazole) and CYP26B1 ( $<10 \mathrm{nM}$ for talarozole and $0.59 \mu \mathrm{M}$ for ketoconazole) (Thatcher et al., 2011; Diaz et al., 2016; Foti et al., 2016a).

Homology Model of CYP26C1 and Molecular Docking of Retinoic Acid Isomers. The experimental data of substrate selectivity, metabolic specificity, ligand binding, and CRABP interactions suggest that CYP26C1 has functional differences compared with CYP26A1 and CYP26B1, and that substrates have different orientations in the CYP26C1 active site compared with other CYP26 enzymes. To investigate the basis of substrate recognition by CYP26C1, a homology model of CYP26C1 was developed and refined by energy minimization and MD simulations (Supplemental Fig. 2; Supplemental Tables 3 and 4). RA isomers (atRA, 9-cis-RA, 13-cis-RA) and 4-oxo-atRA were then docked in silico to the CYP26C1 active site. On the basis of the docking simulations, atRA, 9-cis-RA, and 13-cis-RA all positioned in a similar fashion within the CYP26C1 active site, with the $\beta$-ionone ring closest to the heme iron. 4-oxo-at RA was rotated about $90^{\circ}$ relative to RA isomers in its preferred orientation within the active site (Fig. 11). The docking simulations could appropriately predict the sites of oxidation of all four CYP26C1 substrates. According to the distance between the heme and carbons at positions 4,16 , and 18 on the $\beta$-ionone ring, the CYP26C1 model predicted that atRA, 9-cis-RA, and 13-cis-RA are primarily oxidized at carbon 4, and 4-oxo-atRA is mainly oxidized at carbon 16 (Table 2). At the prochiral carbon 4 position of atRA, the hydrogen that would account for the formation of $4(R)-\mathrm{OH}$ atRA oriented toward the heme iron with a closer distance (3.9 ̊) compared with the hydrogen that, when abstracted, would lead to the formation of $4(S)-\mathrm{OH}-a t$ RA (5.0 $\AA$; Fig. 11A). Thus, the model predicted that CYP26C1 preferentially forms $4(R)$ - over $4(S)-\mathrm{OH}$-atRA from atRA. For 13-cis-RA, pro-S and pro-R hydrogen atoms at C4 position were 3.7 and $3.8 \AA$ away from the heme iron, respectively, leading to the prediction of racemic 4-OH-13-cis-RA formation by CYP26C1 (Fig. 11B). Because the $\beta$-ionone rings of atRA and 9-cis-RA are flipped $180^{\circ}$ relative to each other, the formation of $4(S)-\mathrm{OH}-9$-cis-RA was more favorable than the formation of $4(R)-\mathrm{OH}-9$-cis-RA (Fig. 11C). The distance between the heme iron and the hydrogen atom at the pro-S and pro-R position of 9-cis-RA were 3.1 and $4.6 \AA$, respectively.

Compared with the crystal structure of atRA-bound CYP120A1 (Kühnel et al., 2008), the CYP26C1 model revealed that a C-terminal loop (amino acid L484-L504) occludes the binding pocket occupied by the nonatetraene chain of atRA bound to CYP120A1, forcing atRA to occupy a distinct binding pocket in CYP26C1 (Fig. 10). The amino acids proposed to interact with RA isomers in the active site are shown in Fig. 12 and Supplemental Table 5. Leu-221 and Phe-222 are predicted to interact with the carboxylic acid tail of atRA, 9-cis$\mathrm{RA}$, and 4-oxo-atRA, whereas Ser-120 is predicted to interact with that of 13-cis-RA. Notably, on the basis of the homology model, CYP26C1 active site lacks the positively charged amino acids such as Arg within the active site to interact with the carboxylic acid moiety in atRA.

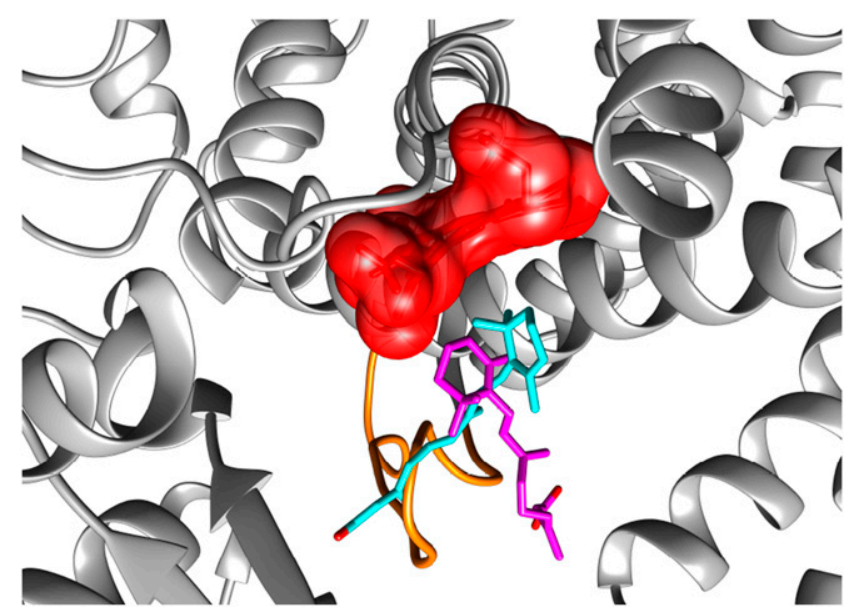

Fig. 10. Docking of atRA to CYP26C1. atRA (magenta) was docked in silico to the homology model of CYP26C1 (gray, heme in red) as described in Materials and Methods. For comparison, the atRA-bound CYP120A1 structure (PDBID: 2VE3) was aligned to our model and the position of the CYP120A1-bound atRA with respect to the CYP26C1 model is shown here (cyan). The C-terminal loop (amino acid L484-L504) that occludes the binding pocket is shown in orange.

\section{Discussion}

To date, most studies related to the CYP26 family have focused on CYP26A1 and CYP26B1, which are known to be important RA hydroxylases (Thatcher and Isoherranen, 2009; Topletz et al., 2012). The importance of CYP26C1 in retinoid clearance has been questioned, as $C y p 26 C 1^{-1-}$ mice do not show any of the phenotypic malformations typical for knockout models of retinoid metabolism (Uehara et al., 2007). However, the tissue-specific importance of the individual CYP26 enzymes in retinoid clearance has not been defined, and depends on both the expression levels of the CYP26 enzymes and their catalytic activity toward the specific substrates. As the sequence homology of CYP26C1 with CYP26A1 and CYP26B1 is low (43\%-51\%), we hypothesized that CYP26C1 differs functionally from CYP26A1 and CYP26B1. Our data demonstrates that CYP26C1 has different ligand (inhibitor and substrate) specificity than CYP26A1 and CYP26B1, illustrated most strongly by the differences in the $\mathrm{IC}_{50}$ values for talarozole and ketoconazole with CYP26 enzymes. Although talarozole and ketoconazole binding to CYP26C1 active site induced a type II binding spectrum with some atypical characteristics similar to CYP26A1 (Thatcher and Isoherranen, 2009), talarozole and ketoconazole $\mathrm{IC}_{50}$ values were more than 100-fold higher with CYP26C1 than with CYP26A1 and CYP26B1 (Thatcher et al., 2011; Diaz et al., 2016). Comparisons of $\mathrm{IC}_{50}$ values between enzymes can be confounded by different substrates and by different substrate concentration $/ K_{\mathrm{m}}$ ratios used, and the inhibitory potency of competitive inhibitors can be underestimated if substrate is used at concentrations approaching its $K_{\mathrm{m}}$. Even still, the $\mathrm{IC}_{50}$ data obtained demonstrate clear differences in ligand interactions within the CYP26 family, and CYP26C1 displays a clear lack of susceptibility to these inhibitors. The different ligand interactions were also evident with retinoids. Whereas atRA has a lower $K_{\mathrm{m}}$ with CYP26A1 and CYP26B1 than 9-cis-RA, with CYP26C1 9-cis-RA exhibited the lowest $K_{\mathrm{m}}$ and highest intrinsic clearance. In addition, the $K_{\mathrm{m}}$ of 9-cis-RA with CYP26C1 $\left(K_{\mathrm{m}}=9.7 \mathrm{nM}\right)$ was much lower than 
TABLE 2

Summary of the predicted orientations of the retinoids within CYP26C1 active site The substrate binding orientation was predicted in silico and the distances of the different carbon atoms were measured to gain insight to regioselectivity of metabolism.

\begin{tabular}{llcccc}
\hline & & \multicolumn{3}{c}{ Distance to Heme Fe $(\AA)$} & \\
\cline { 3 - 5 } Substrate & $\begin{array}{c}\text { Metabolic Site } \\
\text { (Stereoselectivity) }\end{array}$ & $\mathrm{C} 4$ & $\mathrm{C} 16$ & $\mathrm{C} 18$ & $\Delta \mathrm{G}(\mathrm{kcal} / \mathrm{mol})$ \\
\hline atRA & $\mathrm{C} 4(4 R)$ & 4.84 & $>5$ & $>5$ & -8.4 \\
9-cis-RA & $\mathrm{C} 4(4 S)$ & 4.20 & $>5$ & 4.43 & -6.9 \\
13-cis-RA & $\mathrm{C} 4$ (racemic) & 3.93 & $>5$ & $>5$ & -9.5 \\
4-oxo-atRA & C16 & $>5$ & 4.77 & $>5$ & -6.6 \\
\hline
\end{tabular}

with CYP26A1 $\left(K_{\mathrm{m}}=134 \mathrm{nM}\right.$; Thatcher et al., 2011) and CYP26B1 $\left(K_{\mathrm{m}}=555 \mathrm{nM}\right.$; Diaz et al., 2016). This is in agreement with a previous study in which radiolabeled substrate competition assays showed that 9-cis-RA was a better ligand for CYP26C1 than for the other CYP26s (Taimi et al., 2004; Helvig et al., 2011). Interestingly, 9-cis-RA metabolism by CYP26C1 was unaffected by the CRABPs (Fig. 8), possibly because the binding affinity of 9-cis-RA to CRABPs is much weaker than that of atRA and 4-oxo-atRA (Fiorella et al., 1993) resulting in higher free fraction of 9-cis-RA. These findings also indicate a high clearance of 9-cis-RA in vivo, a process unprotected by CRABPs. This may explain the lack of detection of 9-cis-RA in common tissue samples. In human serum 9-cis-RA was detected at a very low level (0.08-0.1 nM) only in a subset of individuals (Arnold et al., 2012). Likewise, in mice 9-cis-RA was only detected in the pancreas (Kane et al., 2010; Kane, 2012). The results shown here suggest that the detection of 9-cis-RA may be dependent on CYP26C1 expression, and further studies are needed to clarify the expression pattern of CYP26C1 in humans, and the role of CYP26C1 in 9-cis-RA clearance. CYP26C1 mRNA has been detected in human fetal liver and brain and in adult human brain, lung, liver, spleen, and testis (Xi and Yang, 2008), suggesting that CYP26C1 is expressed in these retinoid target tissues.

All the data presented here suggest that CYP26C1 plays a unique role in the clearance of the active atRA metabolite 4-oxo-at RA as a 4-oxo-at RA hydroxylase, and that 4-oxo-atRA may be an important active retinoid in humans. However, the microsomal enzyme(s) responsible for 4-oxo-at $\mathrm{RA}$ formation is still unknown. As with CYP26A1 and CYP26B1 (Lutz et al., 2009; Topletz et al., 2012), CYP26C1 forms 4-oxo-atRA from 4-OH-atRA but with minimal efficiency, supporting the prior report that CYP26 enzymes are important in the elimination of bioactive retinoids, not in their formation (Lutz et al., 2009; Topletz et al., 2012). Previously, 4-oxo-atRA has been shown to be a substrate of both CYP26A1 $\left(K_{\mathrm{m}}=63 \mathrm{nM} ; C l_{\text {int }}=91 \mu \mathrm{l} /\right.$ min per picomolar $)$ and CYP26B1 $\left(K_{\mathrm{m}}=29 \mathrm{nM} ; C l_{\text {int }}=15 \mu \mathrm{l} /\right.$ min per picomolar) (Topletz et al., 2012). The $K_{\mathrm{m}}$ values of 4-oxo-atRA with CYP26A1 and CYP26B1 were 4- and 1.8-fold higher than the $K_{\mathrm{m}}$ of 4-oxo-at RA with CYP26C1, respectively, and the $C l_{\text {int }}$ of 4-oxo- $a t \mathrm{RA}$ was 1.7- and 10-fold higher with CYP26C1 than with CYP26A1 and CYP26B1, respectively. Most notably, CYP26C1 appeared to take CRABP-bound 4-oxo-atRA as a substrate, similar to its acceptance of CRABP-bound atRA as a substrate. This suggests that CRABPs play a role in modulating 4-oxo-atRA clearance in the cellular environment. In contrast to our findings, in rat testes microsomes CRABP binding eliminated 4-oxo-at RA metabolism completely (Fiorella and Napoli, 1994), suggesting that the substrate delivery is $\mathrm{P} 450$-specific. This is consistent with the general observation that retinoid exchange between cellular binding proteins and metabolic enzymes is unique to specific binding proteinenzyme pairings (Napoli, 2017). Substrate delivery to CYP26C1 by CRABPs together with the relative expression
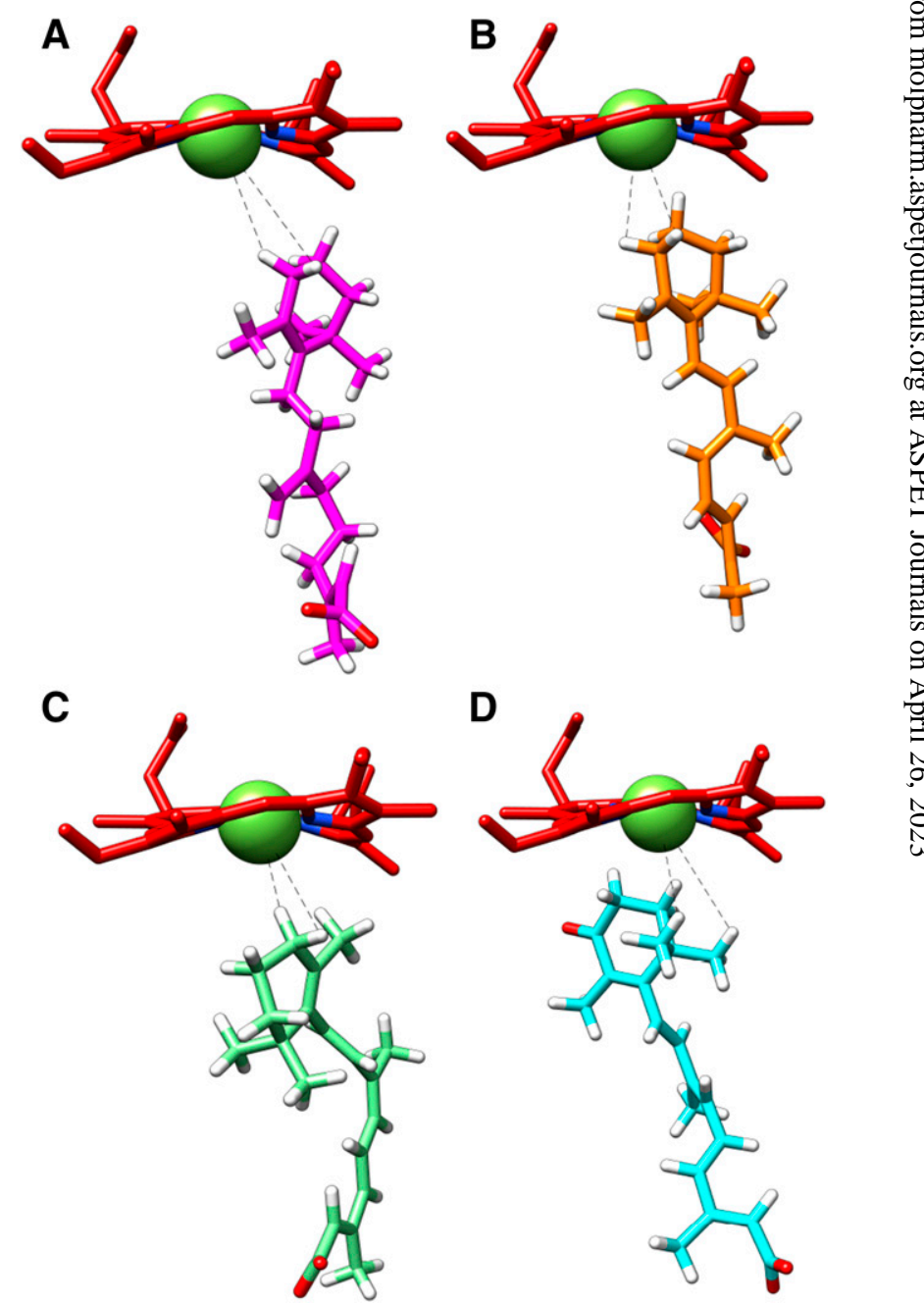

Fig. 11. Comparison of the predicted binding orientations of RA isomers and 4-oxo-atRA with CYP26C1. The predicted binding orientations of atRA [(A), magenta], 13-cis-RA [(B), orange], 9-cis-RA [(C), light green], and 4-oxo-atRA [(D), cyan] with respect to the heme are shown. Dashed lines compare the distances between the heme iron and the hydrogens at $\mathrm{C} 4$ position (A-C) or at C16 position (D). 

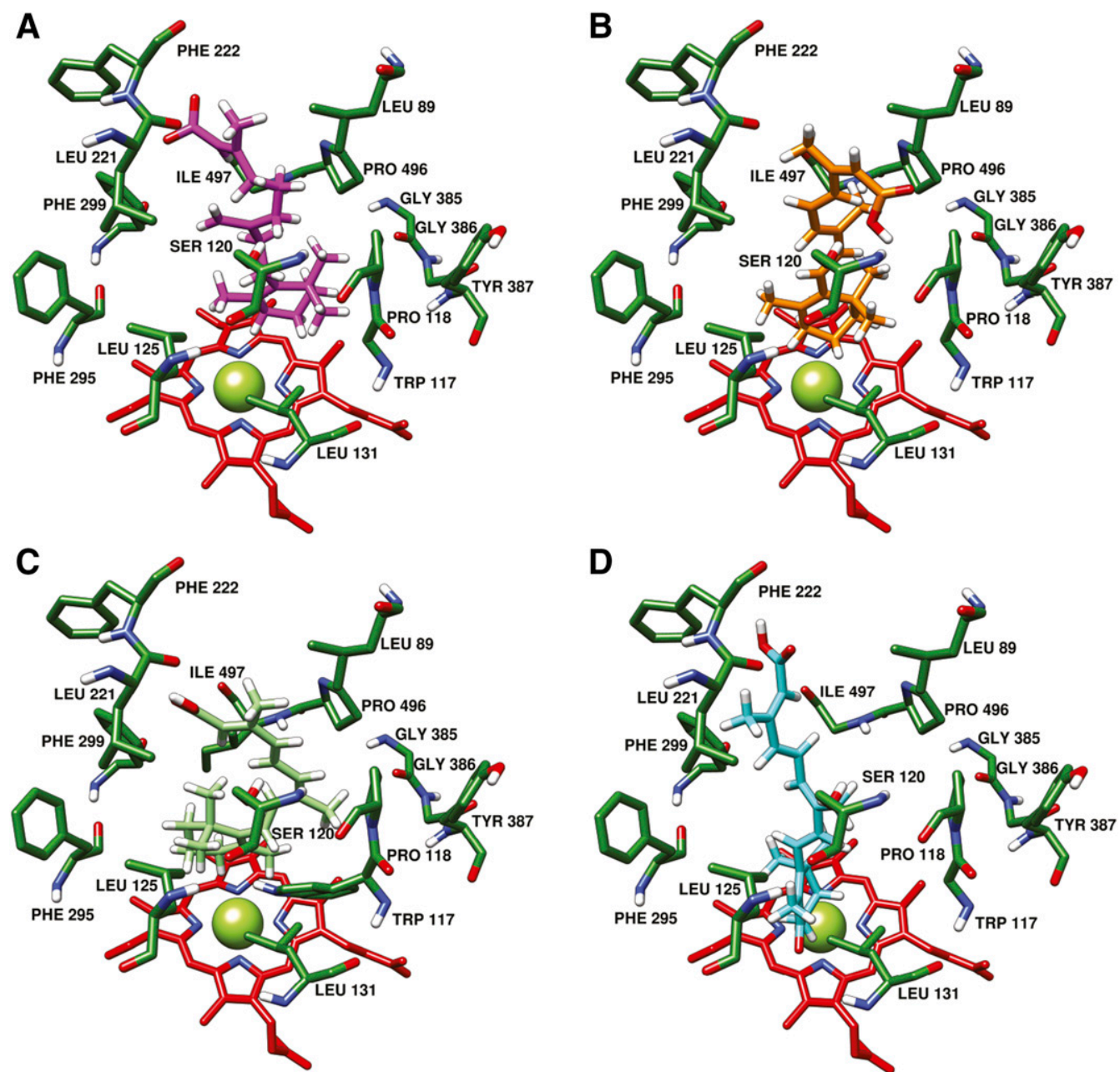

Fig. 12. The active-site residues of CYP26C1 interacting with atRA ((A), magenta), 13-cis-RA ((B), orange), 9-cis-RA ((C), light green) and 4-oxo-atRA ((D), cyan).

levels of CYP26 enzymes, probably determines the physiologic importance of CYP26C1.

The data shown here reveal important information about the P450-CRABP interactions. Owing to the high binding affinity of 4-oxo-atRA and $a t$ RA with CRABPs $\left(K_{\mathrm{d}}<20 \mathrm{nM}\right)$ (Fiorella and Napoli, 1991; Fiorella et al., 1993), 4-oxo-atRA and atRA are expected to be bound with CRABPs in the incubations and in cells with virtually no free retinoids in solution. This lack of free atRA or 4-oxo-atRA in the holoCRABP-containing incubations was confirmed via backpurification of the holo-CRABPs prior to incubations. If CRABPs acted as a sink of retinoids and did not deliver the retinoids for metabolism (i.e., according to free drug hypothesis), the $K_{\mathrm{m}}$ for retinoid metabolism should have been increased in these incubations, as was observed in the presence of albumin (Nelson et al., 2016). In contrast, the $K_{\mathrm{m}}$ values of $a t \mathrm{RA}$ and 4-oxo-atRA bound to CRABP were similar to those of free atRA and 4-oxo-atRA, respectively, with a trend toward lower $k_{\text {cat }}$ with CRABP-bound substrates. These findings are similar to decreased $k_{\text {cat }}$ or $V_{\max }$ and $K_{\mathrm{m}}$ for CRABP-bound atRA reported in rat testis microsomes and recombinant CYP26B1 (Fiorella and Napoli, 1991; Nelson et al., 2016). The data with CYP26C1 supports the notion that CRABPs deliver active retinoids for metabolism via substrate channeling, and strongly suggest that CRABPs deliver both at RA and 4-oxo-at RA to CYP26C1 for metabolism. In contrast, the lack of effect of CRABPs on 9-cis-RA metabolism suggests that CRABPs do not modulate 9-cis-RA clearance. The lack of effect of CRABPs on 9-cis-RA metabolism can be explained by the low binding affinity of 9-cis-RA with CRABPs and the low $K_{\mathrm{m}}$ of 9-cis-RA with CYP26C1.

The differences in substrate specificity, inhibition profiles, and stereospecificity of metabolite formation by CYP26C1, all suggest that the retinoids have different binding orientations within the CYP26C1 active site compared with CYP26A1 and CYP26B1. Specifically, CYP26C1 formed 4-OH-9-cis-RA as the major metabolite of 9-cis-RA and the formation of $16-\mathrm{OH}-$ 9-cis-RA appeared very minor, whereas CYP26A1 made these metabolites at equal levels (Thatcher et al., 2011). Likewise, CYP26C1 formed predominantly the $4(R)-\mathrm{OH}-a t \mathrm{RA}$, whereas CYP26A1 formed the 4(S)-OH-atRA, suggesting different orientation of atRA within the CYP26A1 and CYP26C1 active sites. To explore the binding pocket of CYP26C1, a homology model of CYP26C1 was built on the basis of a complement of P450 crystal structures. Previously, CYP26A1 and CYP26B1 homology models were constructed mainly on the basis of the CYP120A1 crystal structure (Karlsson et al., 2008; Kühnel et al., 2008; Shimshoni et al., 2012; Foti et al., 2016b). The 
predicted orientation of atRA in the active site in these homology models (Shimshoni et al., 2012; Foti et al., 2016b) was similar to that observed in the crystal structure of CYP120A1 (Kühnel et al., 2008), with the carboxylic acid group of atRA interacting with Arg64, Arg86, and Arg90 of CYP26A1 or Trp65, Arg76, Tyr372, and Arg373 of CYP26B1. However, the CYP26C1 homology model revealed that a hairpin loop located at the $\mathrm{C}$ terminus protrudes $\sim 5 \AA$ deeper into the active site than the homologous loop in CYP120A1, which forces atRA and other docked substrates to orient differently, with carboxylic acid interacting with Leu221 and Phe222 (atRA and 9-cis-RA) or Ser120 (13-cis-RA). This orientation was predicted to result in the different stereospecificity of the C4-hydroxylation of atRA. The overall active-site volume of CYP26C1 was predicted to be approximately 200$250 \AA^{3}$, much smaller than the predicted active-site volume of CYP26A1 (918 $\AA^{3}$ ) and CYP26B1 (977 $\AA^{3}$ ) (Foti et al., 2016b). This indicates that the CYP26C1 active site may offer fewer potential substrate binding orientations than do the other two CYP26 enzymes, an observation consistent with the much less diverse metabolite profile observed with CYP26C1 than with CYP26A1 and CYP26B1 (Thatcher et al., 2011; Topletz et al., 2012). The small active-site volume may also explain the high $\mathrm{IC}_{50} \mathrm{~s}$ of talarozole and ketoconazole with CYP26C1. Even bearing in mind the inherent limitations of homology modeling, the distinct structural properties of the CYP26C1 active site predicted by the structural model are consistent with the experimental findings presented here, and successfully captured the regio- and stereospecificity of the enzyme, providing insights into the CYP26C1 active site.

\section{Acknowledgments}

The authors thank Dr. Jay Kirkwood for his skillful assistance on LC-MS/MS experiments and Brian Buttrick for his help in cloning of the CYP26C1.

\section{Authorship Contributions}

Participated in research design: Zhong, Ortiz, Nath, Isoherranen. Conducted experiments: Zhong, Ortiz.

Contributed new reagents or analytic tools: Zelter.

Wrote or contributed to the writing of the manuscript: Zhong, Ortiz, Zelter, Nath, Isoherranen.

\section{References}

Allenby G, Bocquet MT, Saunders M, Kazmer S, Speck J, Rosenberger M, Lovey A, Kastner P, Grippo JF, Chambon P, et al. (1993) Retinoic acid receptors and retinoid $\mathrm{X}$ receptors: interactions with endogenous retinoic acids. Proc Natl Acad Sci USA 90:30-34.

Allenby G, Janocha R, Kazmer S, Speck J, Grippo JF, and Levin AA (1994) Binding of 9-cis-retinoic acid and all-trans-retinoic acid to retinoic acid receptors alpha, beta, and gamma. Retinoic acid receptor gamma binds all-trans-retinoic acid preferentially over 9-cis-retinoic acid. J Biol Chem 269:16689-16695.

Arnold SLM, Amory JK, Walsh TJ, and Isoherranen N (2012) A sensitive and specific method for measurement of multiple retinoids in human serum with UHPLC-MS/ MS. J Lipid Res 53:587-598.

Aström A, Pettersson U, Krust A, Chambon P, and Voorhees JJ (1990) Retinoic acid and synthetic analogs differentially activate retinoic acid receptor dependent transcription. Biochem Biophys Res Commun 173:339-345.

Autenrieth F, Tajkhorshid E, Baudry J, and Luthey-Schulten Z (2004) Classical force field parameters for the heme prosthetic group of cytochrome c. J Comput Chem 25:1613-1622.

Bankson DD, Russell RM, and Sadowski JA (1986) Determination of retinyl esters and retinol in serum or plasma by normal-phase liquid chromatography: method and applications. Clin Chem 32:35-40.

Chambon $\mathrm{P}$ (1996) A decade of molecular biology of retinoic acid receptors. FASEB $J$ 10:940-954.

Diaz P, Huang W, Keyari CM, Buttrick B, Price L, Guilloteau N, Tripathy S, Sperandio VG, Fronczek FR, Astruc-Diaz F, et al. (2016) Development and characterization of novel and selective inhibitors of cytochrome P450 CYP26A1, the human liver retinoic acid hydroxylase. J Med Chem 59:2579-2595.
Durrant JD, Votapka L, Sørensen J, and Amaro RE (2014) POVME 2.0: an enhanced tool for determining pocket shape and volume characteristics. $J$ Chem Theory Comput 10:5047-5056.

Eisenberg D, Lüthy R, and Bowie JU (1997) VERIFY3D: assessment of protein models with three-dimensional profiles. Methods Enzymol 277:396-404.

Fiorella PD, Giguère V, and Napoli JL (1993) Expression of cellular retinoic acidbinding protein (type II) in Escherichia coli. Characterization and comparison to cellular retinoic acid-binding protein (type I). J Biol Chem 268:21545-21552.

Fiorella PD and Napoli JL (1991) Expression of cellular retinoic acid binding protein (CRABP) in Escherichia coli. Characterization and evidence that holo-CRABP is a substrate in retinoic acid metabolism. J Biol Chem 266:16572-16579.

Fiorella PD and Napoli JL (1994) Microsomal retinoic acid metabolism. Effects of cellular retinoic acid-binding protein (type I) and C18-hydroxylation as an initial step. J Biol Chem 269:10538-10544.

Foti RS, Diaz P, and Douguet D (2016a) Comparison of the ligand binding site of CYP2C8 with CYP26A1 and CYP26B1: a structural basis for the identification of new inhibitors of the retinoic acid hydroxylases. J Enzyme Inhib Med Chem 31 (Suppl 2):148-161.

Foti RS, Isoherranen N, Zelter A, Dickmann LJ, Buttrick BR, Diaz P, and Douguet D (2016b) Identification of tazarotenic acid as the first xenobiotic substrate of human retinoic acid hydroxylase CYP26A1 and CYP26B1. J Pharmacol Exp Ther 357: 281-292.

Helvig C, Taimi M, Cameron D, Jones G, and Petkovich M (2011) Functional properties and substrate characterization of human CYP26A1, CYP26B1, and CYP26C1 expressed by recombinant baculovirus in insect cells. J Pharmacol Toxicol Methods 64:258-263.

Herrmann K (1995) Teratogenic effects of retinoic acid and related substances on the early development of the zebrafish (Brachydanio rerio) as assessed by a novel scoring system. Toxicol In Vitro 9:267-283.

Heyman RA, Mangelsdorf DJ, Dyck JA, Stein RB, Eichele G, Evans RM, and Thaller $\mathrm{C}$ (1992) 9-cis retinoic acid is a high affinity ligand for the retinoid X receptor. Cell 68:397-406.

Idres N, Marill J, Flexor MA, and Chabot GG (2002) Activation of retinoic acid receptor-dependent transcription by all-trans-retinoic acid metabolites and isomers. J Biol Chem 277:31491-31498.

Kane MA (2012) Analysis, occurrence, and function of 9-cis-retinoic acid. Biochim Biophys Acta 1821:10-20.

Kane MA, Folias AE, Pingitore A, Perri M, Obrochta KM, Krois CR, Cione E, Ryu JY, and Napoli JL (2010) Identification of 9-cis-retinoic acid as a pancreas-specific autacoid that attenuates glucose-stimulated insulin secretion. Proc Natl Acad Sci USA 107:21884-21889.

Karlsson M, Strid Å, Sirsjö A, and Eriksson LA (2008) Homology models and molecular modeling of human retinoic acid metabolizing enzymes cytochrome P450 26A1 (CYP26A1) and P450 26B1 (CYP26B1). J Chem Theory Comput 4:1021-1027. Kedishvili NY (2016) Retinoic acid synthesis and degradation. Subcell Biochem 81:127-161. Kraft JC and Juchau MR (1993) 9-cis-retinoic acid: a direct-acting dysmorphogen. Biochem Pharmacol 46:709-716.

Kühnel K, Ke N, Cryle MJ, Sligar SG, Schuler MA, and Schlichting I (2008) Crystal structures of substrate-free and retinoic acid-bound cyanobacterial cytochrome P450 CYP120A1. Biochemistry 47:6552-6559

Lovell SC, Davis IW, Arendall WB, III, de Bakker PIW, Word JM, Prisant MG, Richardson JS, and Richardson DC (2003) Structure validation by Calpha geometry: phi,psi and Cbeta deviation. Proteins 50:437-450.

Lutz JD, Dixit V, Yeung CK, Dickmann LJ, Zelter A, Thatcher JE, Nelson WL, and Isoherranen N (2009) Expression and functional characterization of cytochrome P450 26A1, a retinoic acid hydroxylase. Biochem Pharmacol 77:258-268.

Maqbool A, Graham-Maar RC, Schall JI, Zemel BS, and Stallings VA (2008) Vitamin A intake and elevated serum retinol levels in children and young adults with cystic fibrosis. J Cyst Fibros 7:137-141.

Mast N, Charvet C, Pikuleva IA, and Stout CD (2010) Structural basis of drug binding to CYP46A1, an enzyme that controls cholesterol turnover in the brain. $J$ Biol Chem 285:31783-31795.

Monk BC, Tomasiak TM, Keniya MV, Huschmann FU, Tyndall JDA, O'Connell JD, III, Cannon RD, McDonald JG, Rodriguez A, Finer-Moore JS, et al. (2014) Architecture of a single membrane spanning cytochrome P450 suggests constraints that orient the catalytic domain relative to a bilayer. Proc Natl Acad Sci USA 111 3865-3870.

Napoli JL (2012) Physiological insights into all-trans-retinoic acid biosynthesis. Biochim Biophys Acta 1821:152-167.

Napoli JL (2017) Cellular retinoid binding-proteins, CRBP, CRABP, FABP5: effects on retinoid metabolism, function and related diseases. Pharmacol Ther 173:19-33.

Nelson CH, Peng CC, Lutz JD, Yeung CK, Zelter A, and Isoherranen N (2016) Direct protein-protein interactions and substrate channeling between cellular retinoic acid binding proteins and CYP26B1. FEBS Lett 590:2527-2535.

Noy N (2010) Between death and survival: retinoic acid in regulation of apoptosis. Annu Rev Nutr 30:201-217.

Oda A, Yamaotsu N, and Hirono S (2005) New AMBER force field parameters of heme iron for cytochrome P450s determined by quantum chemical calculations of simplified models. J Comput Chem 26:818-826.

Omura T and Sato R (1964) The carbon monoxide-binding pigment of liver microsomes. I. Evidence for its hemoprotein nature. J Biol Chem 239:2370-2378.

Österberg F, Morris GM, Sanner MF, Olson AJ, and Goodsell DS (2002) Automated docking to multiple target structures: incorporation of protein mobility and structural water heterogeneity in AutoDock. Proteins 46:34-40.

Pijnappel WW, Hendriks HF, Folkers GE, van den Brink CE, Dekker EJ, Edelenbosch C, van der Saag PT, and Durston AJ (1993) The retinoid ligand 4-oxoretinoic acid is a highly active modulator of positional specification. Nature $\mathbf{3 6 6}$ : 340-344.

Roy A, Kucukural A, and Zhang Y (2010) I-TASSER: a unified platform for automated protein structure and function prediction. Nat Protoc 5:725-738. 
Sagatova AA, Keniya MV, Wilson RK, Monk BC, and Tyndall JDA (2015) Structural insights into binding of the antifungal drug fluconazole to Saccharomyces cerevisiae lanosterol 14 $\alpha$-demethylase. Antimicrob Agents Chemother 59:4982-4989.

Schmitt-Hoffmann AH, Roos B, Sauer J, Schleimer M, Kovācs P, Stoeckel K, and Maares J (2011) Influence of food on the pharmacokinetics of oral alitretinoin (9-cis retinoic acid). Clin Exp Dermatol 36 (Suppl 2):18-23.

Shahrokh K, Orendt A, Yost GS, and Cheatham TE, III (2012) Quantum mechanically derived AMBER-compatible heme parameters for various states of the cytochrome P450 catalytic cycle. J Comput Chem 33:119-133.

Shimshoni JA, Roberts AG, Scian M, Topletz AR, Blankert SA, Halpert JR, Nelson WL, and Isoherranen N (2012) Stereoselective formation and metabolism of 4-hydroxy-retinoic Acid enantiomers by cytochrome p450 enzymes. J Biol Chem 287:42223-42232.

Stevison F, Jing J, Tripathy S, and Isoherranen N (2015) Role of retinoic acid-metabolizing cytochrome P450s, CYP26, in inflammation and cancer. Adv Pharmacol 74:373-412

Taimi M, Helvig C, Wisniewski J, Ramshaw H, White J, Amad M, Korczak B, and Petkovich M (2004) A novel human cytochrome P450, CYP26C1, involved in metabolism of 9-cis and all-trans isomers of retinoic acid. J Biol Chem 279:77-85.

Tang X-H and Gudas LJ (2011) Retinoids, retinoic acid receptors, and cancer. Annu Rev Pathol 6:345-364.

Thatcher JE, Buttrick B, Shaffer SA, Shimshoni JA, Goodlett DR, Nelson WL, and Isoherranen N (2011) Substrate specificity and ligand interactions of CYP26A1, the human liver retinoic acid hydroxylase. Mol Pharmacol 80:228-239.

Thatcher JE and Isoherranen N (2009) The role of CYP26 enzymes in retinoic acid clearance. Expert Opin Drug Metab Toxicol 5:875-886.

Thatcher JE, Zelter A, and Isoherranen N (2010) The relative importance of CYP26A1 in hepatic clearance of all-trans retinoic acid. Biochem Pharmacol 80:903-912.

Topletz AR, Thatcher JE, Zelter A, Lutz JD, Tay S, Nelson WL, and Isoherranen N (2012) Comparison of the function and expression of CYP26A1 and CYP26B1, the two retinoic acid hydroxylases. Biochem Pharmacol 83:149-163.
Topletz AR, Tripathy S, Foti RS, Shimshoni JA, Nelson WL, and Isoherranen N (2015) Induction of CYP26A1 by metabolites of retinoic acid: evidence that CYP26A1 is an important enzyme in the elimination of active retinoids. $\mathrm{Mol}$ Pharmacol 87:430-441.

Uehara M, Yashiro K, Mamiya S, Nishino J, Chambon P, Dolle P, and Sakai Y (2007) CYP26A1 and CYP26C1 cooperatively regulate anterior-posterior patterning of the developing brain and the production of migratory cranial neural crest cells in the mouse. Dev Biol 302:399-411.

Van Der Spoel D, Lindahl E, Hess B, Groenhof G, Mark AE, and Berendsen HJC (2005) GROMACS: fast, flexible, and free. J Comput Chem 26:1701-1718.

Veal GJ, Cole M, Errington J, Pearson ADJ, Foot ABM, Whyman G, and Boddy AV; UKCCSG Pharmacology Working Group (2007) Pharmacokinetics and metabolism of 13-cis-retinoic acid (isotretinoin) in children with high-risk neuroblastoma - a study of the United Kingdom children's cancer study group. Br J Cancer 96 424-431.

Willhite CC, Hill RM, and Irving DW (1986) Isotretinoin-induced craniofacial malformations in humans and hamsters. J Craniofac Genet Dev Biol Suppl 2:193-209.

Woods CM, Fernandez C, Kunze KL, and Atkins WM (2011) Allosteric activation of cytochrome P450 3A4 by $\alpha$-naphthoflavone: branch point regulation revealed by isotope dilution analysis. Biochemistry 50:10041-10051.

Xi J and Yang Z (2008) Expression of RALDHs (ALDH1As) and CYP26s in human tissues and during the neural differentiation of P19 embryonal carcinoma stem cell. Gene Expr Patterns 8:438-442.

Address correspondence to: Dr. Nina Isoherranen, Department of Pharmaceutics, School of Pharmacy, University of Washington, Health Science Building, Room H-272M, Box 357610, Seattle, WA 98195-7610. E-mail: ni2@ uw.edu 